\title{
THOMAS WILLIAM KÖRNER On the representation of functions by trigonometric series
}

Annales de la faculté des sciences de Toulouse $6^{e}$ série, tome S5 (1996), p. 77-119

<http://www.numdam.org/item?id=AFST_1996_6_S5_77_0>

(C) Université Paul Sabatier, 1996, tous droits réservés.

L'accès aux archives de la revue «Annales de la faculté des sciences de Toulouse » (http://picard.ups-tlse.fr/ annales/) implique l'accord avec les conditions générales d'utilisation (http://www.numdam.org/conditions). Toute utilisation commerciale ou impression systématique est constitutive d'une infraction pénale. Toute copie ou impression de ce fichier doit contenir la présente mention de copyright.

\section{Numdam}

Article numérisé dans le cadre du programme

Numérisation de documents anciens mathématiques

http://www.numdam.org/ 


\title{
On the representation of functions by trigonometric series
}

\author{
Thomas William KöRneR ${ }^{(1)}$
}

ABstract. - We give an account of many of Menšov's theorems on the representation of functions by trigonometric series.

\section{Introduction}

We work on the circle $\mathbb{R} / 2 \pi \mathbb{Z}$. Fourier analysis begins with the formula

$$
f(t) \sim \sum_{r=-\infty}^{\infty} \hat{f}(r) \exp (i r t) .
$$

In his thesis Riemann turned attention to the more general formula

$$
f(t) \sim \sum_{r=-\infty}^{\infty} a_{r} \exp (\text { irt }) .
$$

With the invention of Lebesgue measure it became natural to interpret $\sim$ so that the formula became

$$
\sum_{r=-N}^{N} a_{r} \exp (\text { irt }) \rightarrow f(t)
$$

almost everywhere as $N \rightarrow \infty$, or, more briefly,

$$
\sum_{r=-\infty}^{\infty} a_{r} \exp (i r t)=f(t)
$$

almost everywhere.

(1) Trinity Hall, Cambridge, England twk@pmms.cam.ac.uk 
There are now two natural questions to ask.

Question A (Uniqueness)

If $\sum_{r=-\infty}^{\infty} a_{r} \exp ($ irt $)=0$ almost everywhere does it follow that $a_{r}=0$ for all $r$ ?

\section{Question B (Existence)}

If $f$ is measurable do there exist $a_{r} \in \mathbb{C}$ such that $\sum_{r=-\infty}^{\infty} a_{r} \exp ($ irt $)=$ $f(t)$ ?

Here and elsewhere "measurable" and similar terms refer to Lebesgue measure unless we specify otherwise.

The surprising discovery by Menšov in 1916 that the answer to Question A was no, initiated a long chapter in harmonic analysis which is not yet closed. In 1940, Menšov showed that the answer to Question B is yes, and over the next few years he developed this theme in some remarkable ways. The papers containing his results are long and have a reputation for difficulty. In [6] and [7] I have developed a different approach which I believe to be easier. This paper gives me the opportunity of presenting a coherent treatment along these lines and adding a discussion of what Menšov calls limits of indetermination.

Let us write $S_{n}(f, x)=\sum_{r=-n}^{n} \hat{f}(r) \exp ($ irt $)$ and, if $E$ is measurable, let us write $|E|$ for the Haar measure of $E$ (that is Lebesgue measure normalised so that $|\mathbb{T}|=1$ ). Our key result on which everything else depends is the following.

Lemam 1. - Given any $\eta>0$ there exists a $K(\eta)$ with the following property. Given any $\epsilon>0$ we can find a positive function $f \in A(\mathbb{T})$ with:

(i) $\hat{f}(0)=1$,

(ii) $|\hat{f}(u)| \leq \epsilon$ for all $u \neq 0$.

(iii) $\left|S_{m}(f, x)\right| \leq K(\eta)$ for all $m$.

(iv) $|\{x: f(x) \neq 0\}|<\eta$.

Using Lemma 1 we can obtain one of Menšov's most notable results.

THEOREM 2.- Given $f: \mathbb{T} \rightarrow \mathbb{C}$ a measurable function and $\epsilon>0$ we can find a continuous function $g: \mathbb{T} \rightarrow \mathbb{C}$ with uniformly convergent Fourier series such that

$$
|\{x: f(x) \neq g(x)\}|<\epsilon .
$$


Menšov made great use of his theorem in obtaining his other results but I shall use a development of Lemma 1 instead.

Lemma 3.- Given any $\eta$ there exists a $K_{1}(\eta)$ with the following property. Given any $\epsilon>0$ we can find a positive measure $\mu$ with support of Lebesgue measure 0 and a closed set $E$ with:

(i) $\hat{\mu}(0)=1$,

(ii) $|\hat{\mu}(u)| \leq \epsilon$ for all $u \neq 0$,

(iii) $\left|S_{n}(\mu, x)\right| \leq K_{1}(\eta)$ for all $n \geq 0$ and all $t \in E$,

(iv) $|\mathbb{T} \backslash E| \leq \eta$,

(v) $\hat{\mu}(u) \rightarrow 0$ as $|u| \rightarrow \infty$,

(vi) $S_{n}(\mu, x) \rightarrow 0$ almost everywhere.

One consequence of this lemma which may be new in [6] is the following parallel to Menšov's Theorem 2.

THEOREM 4. - Given any $f \in L^{1}$ and any $\epsilon>0$ there exists a singular measure $\mu$ with $\|\mu\| \leq \epsilon$ such that

$$
S_{n}(\mu+f, x) \rightarrow f(x)
$$

almost everywhere as $n \rightarrow \infty$.

The form of Theorem 4 is explained by the following observation.

Lemma 5. - If $f \in L^{1}$ and $\rho$ is a measure such that

$$
S_{n}(\rho, x)-f(x)
$$

almost everywhere as $n \rightarrow \infty$, then we can write $\rho=f+\sigma$ where $\sigma$ is a singular measure.

Proof. - By the Radon-Nikodym Theorem we may write $\rho=g+\sigma$ where $g \in L^{1}$ and $\sigma$ is a singular measure. Taking $P_{r}$ to be the Poisson kernel we have

$$
P_{r} * \rho=P_{r} * g+P_{r} * \sigma .
$$

But $P_{r} * g(t) \rightarrow g(t)$ and $P_{r} * \sigma(t)-0$ almost everywhere (see [2, $\S 1.7])$ whilst, since we have a summation method, $P_{r} * \rho(t)-g(t)$ almost everywhere as $r \nearrow 1$. Thus $g=f$ and we are done.

Using Lemma 3 we obtain Menšov`s answer to Question B. 
Theorem 6. - If $f$ is measurable we can find $a_{u} \in \mathbb{C}$ such that

$$
\sum_{u=-n}^{n} a_{u} \exp (i u x) \rightarrow f(x)
$$

almost everywhere as $n \rightarrow \infty$.

We can also prove the following amusing result.

Theorem 7 (Menšov's Universal Series). - There exist $a_{u} \in \mathbb{C}$ such that given any measurable function $f$ there exists a sequence $N(j) \rightarrow \infty$ with

$$
\sum_{u=-N(j)}^{N(j)} a_{u} \exp (\text { iux }) \rightarrow f(x)
$$

almost everywhere.

The proof can be extended to give a more general result also due to Menšov.

THEOREM 8. - Let $\mathcal{E}$ be a set of measurable functions such that whenever $f_{n} \in \mathcal{E}$ and $f_{n} \rightarrow f$ almost everywhere then $f \in \mathcal{E}$. Then there exist $a_{u} \in \mathbb{C}$ with $a_{u} \rightarrow 0$ as $|u| \rightarrow \infty$ such that:

(i) If $f \in \mathcal{E}$ then there exists a sequence $N(j) \rightarrow \infty$ with

$$
\sum_{u=-N(j)}^{N(j)} a_{u} \exp (i u x)-f(x)
$$

almost everywhere;

(ii) if there exists a sequence $N(j) \rightarrow \infty$ with

$$
\sum_{u=-N(j)}^{N(j)} a_{u} \exp (i u x) \rightarrow f(x)
$$

then $f \in \mathcal{E}$. 
The interest of this result appears when we consider the cases when $\mathcal{E}$ is empty, when $\mathcal{E}$ has one member, when $\mathcal{E}$ contains two members and when $\mathcal{E}$ is the set of all measurable functions.

Bari extended Menšov's Theorem 6 as follows.

THEOREM 9.- If $f$ is measurable we can find a continuous function $F$ such that $F^{\prime}=f$ almost everywhere and the result of term-by-term differentiation of the Fourier series

$$
\sum_{u=-n}^{n} i u \hat{F}(u) \exp (i u t) \rightarrow f^{\prime}(t)
$$

almost everywhere as $n \rightarrow \infty$.

We shall prove Bari's theorem and add a result which which may be new in $[6]$.

Lemma 10. - If $f \in L^{1}$ we may take $F$ of bounded variation.

So far we have followed the standard modern convention that measurable implies finite almost everywhere. However Menšov was also interested in functions which are allowed to be infinite on sets of positive measure. It turns out that functions $f: \mathbb{T} \rightarrow \mathbb{C} \cup\{\infty\}$ are not very interesting in this context (see [6, Lemma 1.11]) so we restrict consideration to functions $f: \mathbb{T} \rightarrow \mathbb{R}^{*}$ where $\mathbb{R}^{*}=\mathbb{R} \cup\{-\infty, \infty\}$. In order to obtain real partial sums $\sum_{u=-N}^{N} a_{u} \exp (i u t)$ we need to consider $a_{u}$ with $a_{-u}=a_{u}^{*}$. We shall prove the following result of Menšov.

THEOREM 11. - If $f: \mathbb{T} \rightarrow \mathbb{R}^{*}$ is measurable we can find $a_{u}$ with $a_{-u}=a_{u}^{*}$ such that

$$
\sum_{u=-n}^{n} a_{u} \exp (i u t)-f(t)
$$

in measure.

In a brilliant paper [5] Konjagin has shown what Menšov evidently suspected. 
THEOREM 12. - If $a_{-u}=a_{u}^{*}$ for all $u$ then writing

$$
\begin{aligned}
& E_{+}=\left\{t: \limsup _{n \rightarrow \infty} \sum_{u=-n}^{n} a_{u} \exp (\text { iut })=\infty\right\} \\
& E_{-}=\left\{t: \liminf _{n \rightarrow \infty} \sum_{u=-n}^{n} a_{u} \exp (\text { iut })=-\infty\right\},
\end{aligned}
$$

we have

$$
\left|E_{+} \Delta E_{-}\right|=0
$$

Thus we cannot replace convergence in measure by convergence almost everywhere in Theorem 11

Instead we prove the following variation (which I believe to be new) on a result of Menšov.

LemMA 13. - If $g: \mathbb{T} \rightarrow \mathbb{R}^{*}$ is measurable and $g(t) \geq 0$ everywhere then there is a singular measure $\mu$ with $\|\mu\| \leq 1$ such that

$$
\limsup _{n \rightarrow \infty} \sum_{u=-n}^{n} \hat{\mu}(u) \exp (\text { iut })=-\liminf _{n \rightarrow \infty} \sum_{u=-n}^{n} \hat{\mu}(u) \exp (\text { iut })=g(t)
$$

almost everywhere.

Using results stated earlier, this gives our final theorem of Menšov (the last sentence of the theorem is, I think, new).

THEOREM 14. - If $g_{1}, g_{2}: \mathbb{T} \rightarrow \mathbb{R}$ are measurable, $g_{1}(t) \geq g_{2}(t)$ for all $t$ and $E$ is a measurable set then there exist $a_{u} \in \mathbb{C}$ with $a_{-u}=a_{u}^{*}$ for all $u$ such that

$$
\limsup _{n \rightarrow \infty} \sum_{u=-n}^{n} a_{u} \exp (\text { iut })=g_{1}(t), \quad \liminf _{n \rightarrow \infty} \sum_{u=-n}^{n} a_{u} \exp (\text { iut })=g_{2}(t)
$$

almost everywhere for $t \notin E$ and

$$
\limsup _{n \rightarrow \infty} \sum_{u=-n}^{n} a_{u} \exp (\text { iut })=\infty, \quad \liminf _{n \rightarrow \infty} \sum_{u=-n}^{n} a_{u} \exp (i u t)=-\infty,
$$

almost everywhere for $t \in E$. If $g_{2} \in L^{1}$ we may take $a_{u}=\hat{\mu}(u)$ for some singular measure $\mu$. 
Before proceeding to the main part of the paper I would like to make two points, both of which may already be sufficiently obvious to the reader. The first is that, although at first sight, results like Theorems 2 and 6 seem to give useful properties of trigonometric series, their proofs and their general context show that they are reflections of a deep seated pathology. The second is that, however much I seek to emphasise slight improvements, the picture painted here is of a landscape fully explored by Menšov.

\section{The Main Lemma}

The business of this section is to prove Lemma 1. Our main tool, here and elsewhere, will be simple estimates of the type given in Lemma 15 below.

Recall that we say that $f \in A(\mathbb{T})$ if $\|f\|_{A}=\sum_{n=-\infty}^{\infty}|\hat{f}(n)|<\infty$. If $f \in C(\mathbb{T})$ we write $f_{[N]}(t)=f(N t)$.

\section{LEMMA 15}

(i) If $P$ and $Q$ are trigonometric polynomials with $Q$ of degree at most $M$ then, provided only that $N \geq 2 M+1$

$\left|S_{n}\left(P_{[N]} Q, x\right)\right| \leq 2\|\hat{P}\|_{\infty}\|Q\|_{A}+|Q(x)| \max _{m \geq 0} \sup _{t \in \mathbb{T}}\left|S_{m}(P, t)\right|$,

for all $x \in \mathbb{T}$ and all $n \geq 0$.

Also

$$
\left\|\widehat{P_{[N]}} Q\right\|_{\infty}=\|\hat{P}\|_{\infty}\|Q\|_{\infty}
$$

and, if $r$ is fixed, then $\widehat{P_{N} Q}(r)=\hat{P}(0) \hat{Q}(r)$ for all sufficiently large $N$.

(ii) If $f, g \in A\left(\mathbb{T}^{\mathbb{1}}\right)$ and $\epsilon>0$ then, provided only that $N$ is sufficiently large

$\left|S_{n}\left(f_{[N]} g, x\right)\right| \leq 2\|\hat{f}\|_{\infty}\|g\|_{A}+|g(x)| \max _{m \geq 0} \sup _{t \in \mathbb{T}}\left|S_{m}(f, t)\right|+\epsilon$,

for all $x \in \mathbb{T}$ and all $n \geq 0$.

Also

$$
\left\|\widehat{f_{[N]} g}\right\|_{\infty}=\|\hat{f}\|_{\infty}\|g\|_{\infty}+\epsilon
$$

and. if $r$ is fixed, then $\left|\widehat{P_{[N]}} Q(r)-\hat{P}(0) \hat{Q}(r)\right| \leq \epsilon$ for all sufficiently large $N$. 


\section{Proof}

(i) Observe that

$$
\widehat{P_{[N]}} Q(N u+r)=\hat{P}(u) \dot{Q}(r)
$$

whenever $|r| \leq N / 2$. Thus if $n \geq 0$ and we take $m$ such that $|n-m N| \leq N / 2$ we have

$$
\begin{aligned}
\left|S_{n}\left(P_{[N]} Q, x\right)-Q(x) S_{m}(P, N x)\right| & =\left|S_{n}\left(P_{[N]} Q, x\right)-S_{m N+n}\left(P_{[N]} Q, x\right)\right| \\
& \leq(|\hat{P}(m)|+|\hat{P}(-m)|)\|Q\|_{A}
\end{aligned}
$$

so that

$$
\left|S_{n}\left(P_{[N]} Q, x\right)\right| \leq|Q(x)| \sup _{t \in \mathbb{T}}\left|S_{m}(P, t)\right|+2\|\hat{P}\|_{\infty}\|Q\|_{A} .
$$

The stated results now follow.

(ii) Use the fact that the trigonometric polynomials are dense in $A(\mathbb{T})$.

There is a second kind of scaling that we can do. If $F: \mathbb{R} \rightarrow \mathbb{C}$ and $F(x)=0$ for $|x| \geq \pi$ and $N \geq 1$ we can define $F_{(N)}: \mathbb{T} \rightarrow \mathbb{C}$ by the formula $F_{(N)}(t)=f(N t)$ for $|t| \leq \pi$. Provided that $F$ is sufficiently well behaved, we have, not merely that $F_{(N)} \in A(\mathbb{T})$, but also $\left\|F_{(N)}\right\|_{A} \leq K$ for some $K$ independent of $N$ (see for example Chapter II of [3]). We shall work with a particular $F$ for which this is obvious, but our construction is not dependent on making this choice. If we set

$$
\Delta(x)=\max \left(1-\frac{|x|}{\pi}, 0\right) .
$$

It is easy to check that $\hat{\Delta}_{N}(r) \geq 0$ and so $\left\|\Delta_{(N)}\right\|_{A}=\Delta_{N}(0)=1$. Thus taking

$$
G(x)=2 \Delta(x)-\Delta(x), \quad F(x)=G(2 x-\pi)-G(2 x+\pi)
$$

we have $\left\|G_{(N)}\right\|_{A} \leq 3$ and $\left\|F_{(N)}\right\|_{A} \leq 6$. It may be useful to sketch $G_{(N)}$ and $F_{(N)}$.

We can now begin the construction of the function required by Lemma 1 . 
Lemma 16. - Given integers $M, N^{\prime} \geq 1$ and a real number $\eta>0$ we can find an integer $N^{\prime \prime}>N^{\prime}$ and an $H \in A(\mathbb{T})$ such that:

(i) $|H(t)| \leq 1$ for all $t \in \mathbb{T}$,

(ii) $H(t)=0$ for $|t| \geq \pi / M$,

(iii) $|\{t \in \mathbb{T}: H(t)=-1\}| \geq 1 / 8$,

(iv) $\hat{H}(0)=0$,

(v) $\|H\|_{A} \leq 18$

(vi) $\sum_{|r| \leq N^{\prime}}|\hat{H}(r)| \leq \eta$,

(vii) $\sum_{|r| \geq N^{\prime \prime}}|\hat{H}(r)| \leq \eta$.

In particular

$\left|S_{n}(H, t)\right| \leq \begin{cases}18 & \text { for all } n \geq 0 \text { and all } t \\ 1+\eta & \text { for all } n \text { with } n \geq N^{\prime \prime} \text { or } n \leq N^{\prime} \text { and }|t|<\pi / M \\ \eta & \text { for all } n \text { with } n \geq N^{\prime \prime} \text { or } n \leq N^{\prime} \text { and }|t| \geq \pi / M\end{cases}$

Proof. - Consider

$$
H(t)=H_{N}(t)=F_{[8 M N]}(t) G_{M}(t) .
$$

Conditions (i), (ii), (iii), (iv) and (v) are immediate from the construction. (To see (iv) observe that $H$ is odd, to see (v) observe that $\|H\|_{A} \leq$ $\left\|F_{[8 M N]}\right\|_{A}\left\|G_{M}\right\|_{A}$.) Since $F$ is odd, $\hat{F}(0)=0$ and so, for each fixed $r$, $\hat{H}_{N}(r) \rightarrow 0$ as $N \rightarrow \infty$. Thus (vi) will hold for sufficiently large $N$. Once $N$ is fixed, condition (v) shows that (vii) will hold provided only that $N^{\prime \prime}$ is chosen large enough.

The statements about $S_{n}(H, t)$ are direct consequences of conditions (v), (vi), (vii), (i) and (ii).

Lemma 17. - Given any $\epsilon>0$ we can find an $h \in A(\mathbb{T})$ :

(i) $\hat{h}(0)=0$,

(ii) $|\hat{h}(u)| \leq \epsilon$ for all $u$,

(iii) $\left|S_{n}(h, t)\right| \leq 20$ for all $n$,

(iv) $|\{t: h(t) \neq-1\}| \leq 7 / 8$,

(v) $0 \leq h(t) \leq 2$ for all $t$. 
Proof. - Let $\eta>0$ be a real number and let $M \geq 1$ be an integer, both to be chosen later. By the previous lemma we may find $1=N_{0}<N_{1}<$ $\ldots<N(M)$ and $H_{j} \in A(\mathbb{T})$ such that:

(i) $j_{j}\left|H_{j}(t)\right| \leq 1$ for all $t \in \mathbb{T}$,

(ii) ${ }_{j} H_{j}\left(t_{j}\right)=0$ for $|t| \geq \pi / M$,

(iii) $)_{j}\left|\left\{t \in \mathbb{T}: H_{j}(t)=-1\right\}\right| \geq 1 / 8|\mathbb{T}|$,

(iv) ${ }_{j} \hat{H}_{j}(0)=0$,

(v) $)_{j}\left\|H_{j}\right\|_{A} \leq 18$,

(vi) $\sum_{|r| \leq N(j-1)}\left|\hat{H}_{j}(r)\right| \leq \eta$,

(vii) $\sum_{|r| \geq N(j)}\left|\hat{H}_{j}(r)\right| \leq \eta$,

and so, in particular,

$$
\left|S_{n}\left(H_{j}, t\right)\right| \leq \begin{cases}18 & \text { for all } n \geq 0 \text { and all } t \\ 1+\eta & \text { for all } n \text { with } n \geq N(j) \text { or } n \leq N(j-1) \\ & \text { and }|t|<\pi / M \\ \eta & \text { for all } n \text { with } n \geq N(j) \text { or } n \leq N(j) \\ & \text { and }|t| \geq \pi / M .\end{cases}
$$

If we now set

$$
h(t)=\sum_{j=1}^{M} H_{j}\left(t+\frac{2 \pi j}{M}\right),
$$

then conditions (i) and (iv) follow at once from (i) $)_{j},(\text { ii })_{j}$ and $(\text { iv })_{j}$. Conditions (i) $j$ and (ii) $j$ also show that

$$
\left|\hat{H}_{j}(r)\right| \leq\left\|H_{j}\right\|_{1}=\frac{1}{M}
$$

whilst conditions (vi) ${ }_{j}$ and (vii) ${ }_{j}$ show that

$$
\left|\hat{H}_{j}(r)\right| \leq \eta
$$

if $|r| \leq N(j-1)$ or $|r| \geq N(j)$. Thus

$$
|\hat{h}(r)| \leq \sum_{j=1}^{M}\left|\hat{H}_{j}(r)\right|<M \eta+\frac{1}{M}
$$


On the representation of functions by trigonometric series

and condition (iii) follows provided only that we choose $M>2 \epsilon^{-1}$ and $\eta<\epsilon /(2 M)$. Similarly

$$
\left|S_{n}(h, t)\right| \leq \sum_{j=1}^{M}\left|S_{n}\left(H_{j}, t+\frac{2 \pi j}{M}\right)\right| \leq 1+M \eta+18
$$

for all $t$ and all $n$, so that condition (iv) holds provided only that we choose $\eta<1 / M$.

A trivial modification gives the next result.

Lemma 18. - Given any $\epsilon>0$ we can find an $f \in A(\mathbb{T})$ :

(i) $\hat{f}(0)=1$,

(ii) $|\hat{f}(u)| \leq \epsilon$ for all $u \neq 0$,

(iii) $\left|S_{n}(f, t)\right| \leq 21$ for all $n$,

(iv) $|\{t: f(t) \neq 0\}| \leq 7 / 8$,

(v) $0 \leq f(t) \leq 2$ for all $t$.

Proof. - Set $f=h+1$ in Lemma 17 .

We now "concentrate" the function $f$ of Lemma 18 by repeated multiplication.

Lemma 19.- Given any $\delta>0$ we can find a sequence of functions $f_{n} \in A(\mathbb{T})$, with $f_{0}=1$, and closed sets

$$
\emptyset=E_{0} \subseteq E_{1} \subseteq E_{2} \ldots
$$

such that. if $n \geq 1$,

(i) $n\left|\hat{f}_{n}(u)-\hat{f}_{n-1}(u)\right| \leq \delta 2^{-(n+1)}$ for all $u$,

(ii) $n\left|S_{m}\left(f_{n}, t\right)\right| \leq 2^{r-1} 25-2^{-n}$

for all $m \geq 0$, all $t \in E_{r}$ and all $1 \leq r \leq n-1$,

(iii) $)_{n}\left|S_{m}\left(f_{n}, t\right)\right| \leq 2^{n-1} 25-2^{-n}$ for all $m \geq 0$, and all $t$,

(iv) $f_{n}(t)=0$ for $t \in E_{n}$,

(v) $n \quad\left|\mathbb{T} \backslash E_{n}\right| \leq(15 / 16)^{n}$,

(vi) ${ }_{n} 2^{n} \geq f_{n}(t) \geq 0$ for all $t$,

(vii) $n$ supp $f_{n} \subseteq \operatorname{supp} f_{n-1}$. 
Proof. - We start the induction by setting $f_{1}=f$ and $E_{1}=f^{-1}(0)$ where $f$ is the function of Lemma 18. Provided that $\epsilon$ is chosen small enough, conditions (i) $)_{1}$ to (vii) $)_{1}$ are automatically satisfied.

Now suppose that we have constructed $f_{j}$ and $E_{j}$ for $1 \leq j \leq n$. We set $f_{n+1}(t)=f_{n}(t) f(N t)$ where $f$ is the function of Lemma 18 with an $\epsilon>0$ and an integer $N \geq 1$ to be chosen later. If we set

$$
E_{n+1}=E_{n} \cup\{t: f(N t)=0\}
$$

then conditions $(\mathrm{v})_{n+1},(\mathrm{vi})_{n+1}$ and (vii $)_{n+1}$ follow automatically from conditions $(\mathrm{v})_{n}$ and $(\mathrm{vi})_{n}$ combined with condition (v) of Lemma 18. Condition (iv) $)_{n+1}$ follows from (iv) $n$ and condition (iv) of Lemma 18 provided only that $N$ is large enough.

To prove the remaining conditions set $g=f-1$ and $g_{n+1}(t)=$ $f_{n}(t) g(N t)=f_{n}(t) g_{[N]}(t)$. We observe that

$$
f_{n+1}-f_{n}=g_{n+1} \text {. }
$$

Since

$$
\left\|\hat{g}_{n+1}\right\|_{\infty}=\left\|\widehat{f_{n}} \widehat{g_{[N]}}\right\|_{\infty} \leq\left\|\widehat{g}_{[N]}\right\|_{\infty}\left\|f_{n}\right\|_{A}=\|\hat{g}\|_{\infty}\left\|f_{n}\right\|_{A}=\epsilon\left\|g_{n}\right\|_{A},
$$

we see that condition $(\mathrm{i})_{n+1}$ will hold provided only that $\epsilon$ is chosen small enough. Further, choosing $M$ sufficiently large that

$$
\sum_{|u| \geq M}\left|\hat{f}_{n}(u)\right| \leq 2^{-n-5}
$$

we know that, provided $\epsilon$ is small enough

$$
\sum_{|u| \leq M}\left|\hat{g}_{n+1}(u)\right| \leq 2^{-n-5}
$$

and so

$$
\begin{aligned}
\left|S_{m}\left(f_{n+1}, t\right)\right| & \leq\left|S_{m}\left(f_{n}, t\right)\right|+\mid S_{m}\left(g_{n+1}, t\right) \\
& \leq \max \left(\left|S_{m}\left(f_{n}, t\right)\right|,\left|f_{n}(t)\right|+\left|S_{m}\left(g_{n+1}, t\right)\right|\right)+2^{-n-3} .
\end{aligned}
$$

But, by Lemma 15, we know that, provided only that $N$ is large enough,

$$
\begin{aligned}
\left|S_{m}\left(g_{n+1}, t\right)\right| & =\left|S_{m}\left(g_{[N]} f_{n},\right)\right| \\
& \leq 2\|\hat{g}\|_{\infty}\left\|f_{n}\right\|_{A}+\left|f_{n}(t)\right| \max _{p \geq 0} \sup _{t \in \mathbb{T}}\left|S_{p}(g, t)\right|+2^{-n-5} .
\end{aligned}
$$


Now, by Lemma 18(iii) $\left|S_{p}(g, t)\right|=\left|S_{p}(g, t)+1\right| \leq 23$ so that, provided only that $\epsilon$ is small enough,

$$
\left|S_{m}\left(g_{n+1}, t\right)\right| \leq 23\left|f_{n}(t)\right|+2^{-n-4}
$$

and so

$$
\left|S_{m}\left(f_{n+1}, t\right)\right| \leq \max \left(\left|S_{m}\left(f_{n}, t\right)\right|, 24\left|f_{n}(t)\right|\right)+2^{-n-2},
$$

for all $m \geq 0$ and all $t$. Conditions (ii) $)_{n}$ and (vi) $)_{n}$ now give (ii) $)_{n+1}$ and $(\mathrm{vi})_{n+1}$. The inductive step can thus be completed and the lemma is proved.

The key Lemma 1 is now obvious.

Proof. - Without loss of generality we may suppose $\eta<1 / 100$. Choose $n$ such that $(15 / 16)^{n}|\mathbb{T}|<\eta$ and set $K(\eta)=2^{n} 25$. If we now set $\delta=\eta / 2$ and take $f_{n}$ as in Lemma 19 above then conditions (i) $m$ with $1 \leq m \leq n$ tell us that :

(i) $)^{\prime}\left|\hat{f}_{n}(u)-\hat{1}(u)\right| \leq \delta$ i.e. $\left|\hat{f}_{n}(0)-1\right| \leq \delta$ (so that, in particular, $\hat{f}_{n}(0) \geq 1 / 2$ ) whilst $\left|\hat{f}_{n}(u)\right| \leq \delta$ for all $u \neq 0$.

Condition (iii) $)_{n}$ gives us :

(iii) ${ }^{\prime}\left|S_{m}\left(f_{n}, t\right)\right| \leq 2^{n-1} 25$ for all $m \geq 0$.

Conditions (iv) $)_{n}$ and $(\mathrm{v})_{n}$ tell us that

$$
\text { (iv) })^{\prime}\left|\left\{x: f_{n}(x) \neq 0\right\}\right|<\eta \text {, }
$$
whilst condition (vi) $)_{n}$ tells us that $f_{n}$ is positive.

If we now set $f=\left(\hat{f}_{n}(0)\right)^{-1} f_{n}$ we have $2 f_{n}(t) \geq f(t) \geq 0$ so the conclusions of the lemma may be read off.

We could have proved Lemma 1 directly by modifying the proof of Lemma 18 (this would make a good exercise for the reader) but the inductive concentration argument in Lemma 19 also gives us Lemma 3.

Proof. - Without loss of generality suppose $\epsilon<1$ and set $\delta=\epsilon / 2$. Consider the functions $f_{n}$ of Lemma 19. Let us set $\mathrm{d} \mu_{n}=f_{n}(x)(2 \pi)^{-1} \mathrm{~d} x$ 
(so that $\mu_{n}$ is the "obvious" measure associated with $f_{n}$ ). By condition (vii) $)_{n}, \mu_{n}$ is a positive measure. We note that (i) $)_{n}$ becomes

$$
\left|\hat{\mu}_{n}(u)-\hat{\mu}_{n-1}(u)\right| \leq \delta 2^{-(n+1)}
$$

for all $u$. In particular $\left\|\mu_{n}\right\|=\hat{\mu}_{n}(0)$ is bounded and so, $\mu_{n}$ converges weakly to a positive measure $\mu^{\prime}$ with

$$
\left|\hat{\mu}^{\prime}(u)-\hat{\mu}_{0}(u)\right| \leq \delta .
$$

If we take $\delta=1 / 2$, then, since $\hat{\mu}_{0}(0)=1$ we have $3 / 2 \geq\left\|\mu^{\prime}\right\| \geq 1 / 2$. We now set $\mu=\left\|\mu^{\prime}\right\|^{-1} \mu^{\prime}$. Since $\hat{\mu}_{0}(u)=0$ for all $u \neq 0$, we have

$$
|\hat{\mu}(u)| \leq 2\left|\hat{\mu}^{\prime}(u)\right| \leq 2 \delta \leq \epsilon
$$

for all $u \neq 0$.

Condition (vii) $)_{n}$ shows us that

$$
\operatorname{supp} \mu=\operatorname{supp} \mu^{\prime} \subseteq \operatorname{supp} f_{n}
$$

Conditions (iv) $)_{n}$ and $(\mathrm{v})_{n}$ now show that $|\operatorname{supp} \mu|=0$. Condition (ii) $n$ shows that

$$
\left|S_{m}(\mu, t)\right| \leq 2\left|S_{m}\left(\mu^{\prime}, t\right)\right| \leq 2^{r} 25
$$

for all $m \geq 0$, all $t \in E_{r}$. But condition $(\mathrm{v})_{r}$ tells us that

$$
\left|\mathbb{T} \backslash E_{r}\right| \leq\left(\frac{15}{16}\right)^{r}
$$

If $\eta>0$ is given we can choose an $r$ such that $\eta<(15 / 16)^{r}|\mathbb{T}|$. If we now set $K_{1}(\eta)=2^{r} 25$ then conclusions (iii) and (iv) of our lemma follow on setting $E=E_{r}$. Condition (v) follows from the observation that $\hat{\mu}_{n}(u) \rightarrow 0$ as $|u| \rightarrow \infty$. Condition (iv) is a consequence of the general result that if $\rho$ is a measure with $\hat{\rho}(u) \rightarrow 0$ as $|u| \rightarrow \infty$ then $S_{m}(\rho, t) \rightarrow 0$ for all $t \notin \operatorname{supp} \rho$, but it can also be obtained directly by arguments of the type used above.

\section{Consequences}

Many of the results announced in the introduction now follow without much more work. We start with Theorem 2, Menšov's theorem on the adjustment of functions. 
At first sight Lemma 1 looks rather far removed from Theorem 2 but the next lemma shows that they are rather close.

Lemma 20.-Given any $\eta>0$ there exists a $K_{2}(\eta)$ with the following property. Given any trigonometric polynomial $Q$ and any $\kappa>0$ we can find $q \in A(\mathbb{T})$ with:

(i) $\left|S_{n}(q, x)\right| \leq K_{2}(\eta)\|Q\|_{\infty}$ for all $n$ and all $x \in \mathbb{T}$,

(ii) $|\{x: q(x) \neq Q(x)\}|<\eta$,

(iii) $|\hat{q}(u)| \leq \kappa$.

Proof. - Take $K(\eta)$ as in Lemma 1 and set $K_{2}(\eta)=K^{\prime}(\eta)+3$. Take $f$ as in Lemma 1 for some $\epsilon$ still to be determined. We set

$$
q(x)=(1-f(N x)) Q(x) .
$$

By condition (iv) of Lemma 1

$$
|\{x: q(x) \neq Q(x)\}|=|\{x: f(N x) \neq 0\}|=|\{x: f(x) \neq 0\}|<\eta .
$$

Next we observe that conditions (i) and (ii) of Lemma 1 show that $\|\widehat{1-f}\|_{\infty} \leq \epsilon$. Thus Lemma 15 shows that, provided $N$ is large enough (actually $N=2 \times \operatorname{degree}(Q)+1$ will do) and $\epsilon$ small enough, conclusions (ii) and (iii) hold.

Theorem 2 now follows.

Proof. - If $f: \mathbb{T} \rightarrow \mathbb{C}$ is a measurable function and $\epsilon>0$ then. by a theorem of Lusin, we can find a continuous function $F$ such that

$$
|\{x: f(x) \neq F(x)\}|<\frac{\epsilon}{2} .
$$

By Fejér's theorem we can find a sequence of trigonometric polynomials tending uniformly to $F$. By extracting a sufficiently rapidly convergent subsequence we can obtain trigonometric polynomials $Q_{1}, Q_{2}, Q_{3}, \ldots$ such that $\sum_{j=1}^{\infty} Q_{j}$ converges uniformly to $F$ and

$$
\left\|Q_{j}\right\|_{\infty} \leq K_{2}\left(2^{-j} \epsilon\right)^{-1} 2^{-j}
$$

for all $j \geq 2$. 
We set $q_{1}=Q_{1}$ and use Lemma 20 to obtain, for each $j \geq 2, q_{j} \in A(\mathbb{T})$ with:

(i) ${ }_{j}\left|S_{n}\left(q_{j}, x\right)\right| \leq K_{2}\left(2^{-j} \epsilon\right)\left\|Q_{j}\right\|_{\infty} \leq 2^{-j}$ for all $n$ and $x$,

(ii) $j\left|\left\{x: q_{j}(x) \neq Q_{j}(x)\right\}\right|<2^{-j} \epsilon$.

Since $q_{1}, q_{2}, \ldots, q_{m} \in A(\mathbb{T}), S_{n}\left(\sum_{j=1}^{m} q_{j}, \cdot\right)$ converges uniformly to $\sum_{j=1}^{m} q_{j}$. On the other hand conditions (i) ${ }_{j}$ show that

$$
\left\|S_{n}\left(\sum_{j=m+1}^{\infty} q_{j}, \cdot\right)\right\|_{\infty} \leq 2^{-m}
$$

for all $n \geq 0$ and all $m \geq 1$. Thus $g=\sum_{j=1}^{\infty} q_{j}$ has uniformly convergent Fourier series. Condition (ii) $j$ and the definition of $q_{1}$ show that

$$
\left|\left\{x: \sum_{j=1}^{\infty} q_{j}(x) \neq \sum_{j=1}^{\infty} Q_{j}(x)\right\}\right|<\frac{\epsilon}{2} .
$$

In other words

$$
|\{x: g(x) \neq F(x)\}|<\frac{\epsilon}{2}
$$

and, recalling how we chose $F$, we see that the proof is complete.

In the same way as Lemma 1 gave us Lemma 20 so Lemma 3 has the following simple extension.

Lemma 21. - Given any $\eta>0$ there exists a $K_{3}(\eta)$ with the following property. Given any trigonometric polynomial $Q$ and any $\kappa>0$ we can find a measure $\rho$ with support of Lebesgue measure zero and a closed set $L$ such that:

(i) $\left|S_{n}(Q+\rho, x)\right| \leq K_{3}(\eta)|Q(x)|$ for all $n$ and all $x \in L$,

(ii) $|\mathbb{T} \backslash L|<\eta$,

(iii) $\|\rho\| \leq 2\|Q\|_{1}$,

(iv) $\left|(Q+\rho)^{\wedge}(u)\right| \leq \kappa$ for all $u$,

(v) $\hat{\rho}(u) \rightarrow 0$ as $|u| \rightarrow \infty$,

(vi) $S_{n}(Q+\rho, x) \rightarrow Q(x)$ almost everywhere. 
Proof. - Take $K_{1}(\eta)$ as in Lemma 3 and set $K_{3}(\eta)=K_{1}(\eta)+3$. Take $\mu$ and $E$ as in Lemma 1 for some $\epsilon$ still to be determined. If $Q$ has degree $M$ we take $N \geq 2 M+1$ with the value of $N$ to be determined later. We take $\mu_{N}$ to be the measure given by $\hat{\mu}_{N}(r N)=\hat{\mu}(r), \mu_{N}(v)=0$ otherwise, and set

$$
\rho=-Q \mu_{N} .
$$

Conditions (v) and (vi) is follow automatically from conditions (v) and (vi) of Lemma 3 . we observe that

$$
\|\rho\|=\int_{\mathbb{T}}|Q(t)| \mathrm{d}\left|\mu_{N}\right|(t) \rightarrow\|Q\|_{1}\|\mu\|=\|Q\|_{1},
$$

as $N \rightarrow \infty$ so conclusion (iii) holds provided only that we take $N$ large enough. We set

$$
L=E_{N}=\{N x: x \in E\}
$$

so that conclusion (ii) follows from the corresponding result for $E$.

If we set $\nu=1-\mu$ and $\nu_{N}=1-\mu_{N}$ then Lemma 3 shows that:

(ii) $|\hat{\nu}(u)| \leq \epsilon$ for all $u$,

(iii) ${ }^{\prime}\left|S_{n}(\nu, x)\right| \leq K_{1}(\eta)+1$ for all $n \geq 0$ and all $t \in E$.

But the arguments used to prove Lemma 15 show that

$$
\begin{aligned}
\left|S_{n}(Q+\rho, x)\right| & =\left|S_{n}\left(Q \nu_{N}, x\right)\right| \\
& \leq 2\|\hat{\rho}\|_{\infty}\|Q\|_{A}+|Q(x)| \max _{m \geq 0} \sup _{t \in E}\left|S_{m}(\nu, t)\right| \\
& \leq 2 \epsilon\|Q\|_{A}+|Q(x)|\left(K_{1}(\eta)+1\right)
\end{aligned}
$$

for all $x \in L$ and all $n \geq 0$. Condition (i) now follows, provided only that we choose $\epsilon$ small enough. Condition (iv) can be ensured in a similar but simpler manner.

Theorem 4 now follows.

Proof. - By using the theorems of Lusin and Fejér we can find a sequence of trigonometric polynomials $Q_{j}$ and closed sets $H_{j}$ such that:

(a) $\sum_{j=1}^{\infty} Q_{j}$ converges pointwise to $f$ except on a set $E$ of Lebesgue measure zero, 
(b) $\sum_{j=2}^{\infty}\left\|Q_{j}\right\|_{1} \leq \epsilon / 2$,

(c) $\left|Q_{j}(x)\right| \leq K_{3}\left(2^{-j}\right)^{-1} 2^{-j}$ for all $x \in H_{j}$ and all $j \geq 2$,

(d) $\left|H_{j}\right| \leq 2^{-j}$ for $j \geq 2$.

Lemma 21 now tells us that we can find measures $\rho_{j}$ with support of Lebesgue measure zero and measurable sets $E_{j}=\mathbb{T} \backslash L_{j}$ such that, if $j \geq 2$.

(i) $)_{j}\left|S_{n}\left(Q_{j}+\rho_{j}, x\right)\right| \leq K_{3}\left(2^{-j}\right)\left|Q_{j}(x)\right|$ for all $n$ and all $x \notin E_{j}$,

(ii) $j\left|E_{j}\right|<2^{-j}$,

(iii) $)_{j}\left\|\rho_{j}\right\| \leq 2\left\|Q_{j}\right\|_{1}$.

We set $\rho_{1}=0$.

Conditions (b) and (iii) tell us that $\sum_{j=2}^{\infty} \rho_{j}$ converges in measure norm to a measure $\mu$ with $\|\mu\| \leq \epsilon$. Since the $\rho_{j}$ are singular so is $\mu$. Since each of the $\rho_{j}$ has support of Lebesgue zero measure and a standard theorem tells us that

$$
S_{n}\left(\rho_{j}, x\right) \rightarrow 0 \text { for } x \notin \operatorname{supp} \rho_{j}
$$

as $n \rightarrow \infty$ it follows that there exists a set $F_{m}$ of Lebesgue measure 0 such that

$$
S_{n}\left(\sum_{j=1}^{m}\left(Q_{j}+\rho_{j}\right), x\right) \rightarrow \sum_{j=1}^{m} Q_{j}(x) \quad \text { for } x \notin F_{m}
$$

as $n-\infty$. But condition (i) $j_{j}$ combined with condition (c) tells us that, if $j \geq 2$,

$$
\left|S_{n}\left(Q_{j}+\rho_{j}, x\right)\right| \leq 2^{j}
$$

for all $x \notin H_{j}$ and so

$$
\left|S_{n}\left(\sum_{j=m+1}^{\infty}\left(Q_{j}+\rho_{j}\right), x\right)\right| \leq 2^{-m}
$$

for all $x \notin \bigcup_{j=m+1}^{\infty}\left(E_{j} \cup H_{j}\right)$ and all $n \geq 0$. If follows that

$$
S_{n}(f+\mu, x)=S_{n}\left(\sum_{j=1}^{\infty}\left(Q_{j}+\rho_{j}\right), x\right) \rightarrow f(x)
$$


as $n \rightarrow \infty$ for all $x \notin E \cup \bigcap_{m=1}^{\infty} \bigcup_{j=m+1}^{\infty}\left(E_{j} \cup H_{j}\right)$. Since $\left|E_{j} \cup H_{j}\right| \leq 2^{-j+1}$ and $|E|=0$ this tells us that $S_{n}(f+\mu, x) \rightarrow f(x)$ almost everywhere as required.

The proof of Theorem 6 is similar but simpler.

Proof. - By using the theorems of Lusin and Fejér we can find a sequence of trigonometric polynomials $Q_{j}$ and closed sets $H_{j}$ such that:

(a) $\sum_{j=1}^{\infty} Q_{j}$ converges pointwise to $f$ except on a set $E$ of Lebesgue measure zero,

(c) $\left|Q_{j}(x)\right| \leq K_{3}\left(2^{-j}\right)^{-1} 2^{-j}$ for all $x \in H_{j}$ and all $j \geq 2$,

(d) $\left|H_{j}\right| \leq 2^{-j}$ for $j \geq 2$.

Lemma 21 now tells us that we can find measures $\rho_{j}$ with support of Lebesgue measure zero and measurable sets $E_{j}=\mathbb{T} \backslash L_{j}$ such that, if $j \geq 1$.

(i) $)_{j}\left|S_{n}\left(Q_{j}+\rho_{j}, x\right)\right| \leq K_{3}\left(2^{-j}\right)\left|Q_{j}(x)\right|$ for all $n$ and all $x \notin E_{j}$,

(ii) $j\left|E_{j}\right|<2^{-j}$,

(iv) $)_{j}\left|\hat{\rho}_{j}(u)\right| \leq 2^{-j}$.

If we now set $a_{u}=\sum_{j=1}^{\infty} \hat{\rho}_{j}(u)$, the proof that $\sum_{u=-n}^{n} a_{u} \exp (i u t) \rightarrow f(t)$ almost everywhere follows the lines of the previous proof.

\section{Convergence of Subsequences}

Results like the existence of universal trigonometric series can be reduced to exercises in measure theory by means of the following lemma.

LEMMA 22.- Given any sequence $f_{n}$ of continuous functions with $\left\|f_{n}-f_{n-1}\right\|_{\infty} \rightarrow 0$ as $n \rightarrow \infty$ we can find $a_{u} \in \mathbb{C}$ with $a_{u} \rightarrow 0$ as $|u| \rightarrow \infty$ and a sequence of integers $N(j)$ with $0<N(1)<N(2)<\ldots$ such that:

(i) $\sum_{u=-N j}^{N(j)} a_{u} \exp (i u t)-f_{j}(t)-0$ almost everywhere as $j \rightarrow \infty$.

(ii) If $\epsilon>0$ is fixed, then

$$
\begin{aligned}
& \mid\left\{t: \mid \sum_{u=-N}^{N} a_{u} \exp (\text { iut })-f_{j}(t) \mid<\epsilon \text { for all } N(j) \leq N \leq N(j+1)\right\} \mid \rightarrow 1 \\
& \quad \text { as } k \rightarrow 0 .
\end{aligned}
$$


Proof. - Since we can find trigonometric polynomials $P_{n}$ with $\left\|P_{n}-f_{n}\right\|_{\infty} \rightarrow 0$ there is no loss of generality in supposing that the $f_{n}$ are trigonometric polynomials. We set $Q_{0}=f_{0}=0$ and $Q_{j}=f_{j}-f_{j-1}$ for all $j \geq 1$. Since $\left\|Q_{j}\right\|_{\infty} \rightarrow 0$ as $j \rightarrow \infty$ we can find $\eta_{j}>0$ with

$$
K\left(\eta_{j}\right)\left\|Q_{j}\right\|_{\infty} \leq \eta_{j}
$$

for all $j$, where $K_{3}\left(\eta_{j}\right)$ is defined as in Lemma 21, and $\eta_{j} \rightarrow 0$ as $j \rightarrow \infty$.

We now construct inductively a sequence of measures $\tau_{j}$ together with a sequence of integers $N(j)$ as follows. Set $\tau_{0}=0$ and $N(-1)=0$. Suppose now that $N(j-1)$ has been fixed and we have constructed $\tau_{0}, \tau_{1}, \ldots, \tau_{j-1}$ in such a way that:

(i) ${ }_{j} \quad S_{n}\left(\sum_{p=0}^{j-1} \tau_{p}, x\right) \rightarrow f_{j-1}(x)$

almost everywhere as $n \rightarrow \infty$. Simple measure theory tells us that we can find $N(j)>N(j-1)$ and a measurable set $E_{j}$ with

(ii) $j\left|E_{j}\right| \leq 2^{-j}$,

(iii) $j\left|S_{n}\left(\sum_{p=0}^{j-1} \tau_{p}, x\right)-f_{j-1}(x)\right| \leq 2^{-j}$

for all $x \notin E_{j}$ and all $n \geq N(j)$.

Let $\kappa_{j}>0$ be a small number to be determined. By Lemma 21 we can find a measure $\tau_{j}=Q_{j}+\rho_{j}$ and a measurable set $F_{j}=\mathbb{T} \backslash L_{j}$ such that:

$(\mathrm{v})_{j}\left|S_{n}\left(\tau_{j}, x\right)\right| \leq K_{3}\left(\eta_{j}\right)\left\|Q_{j}\right\|_{\infty}$ for all $n$ and all $x \notin F_{j}$,

(vi) $)_{j}\left|F_{j}\right|<\eta_{j}$,

(vii) ${ }_{j}^{\prime}\left|\hat{\tau}_{j}(u)\right| \leq \kappa_{j}$ for all $u$.

(vii) ${ }_{j} \hat{\tau}_{j}(u) \rightarrow 0$ as $|u| \rightarrow \infty$.

$(\mathrm{ix})_{j} S_{n}\left(\tau_{j}, x\right) \rightarrow Q_{j}(x)$ almost everywhere.

By choosing $\kappa_{j}$ small enough, we can ensure that:

$$
\text { (vii) } \sum_{|u| \leq N(j-1)}\left|\hat{\tau}_{j}(u)\right| \leq 2^{-j-1} \text { and }\left|\hat{\tau}_{j}(u)\right| \leq 2^{-j} \text { for all } u \text {. }
$$

Combining (viii) ${ }_{j}$ and (i) ${ }_{j}$ we obtain (i) ${ }_{j+1}$ so we may move onwards to the next stage of the induction. 
On the representation of functions by trigonometric series

Condition (vii) ${ }_{j}$ allows us to set

$$
a_{u}=\sum_{k=0}^{\infty} \hat{\tau}_{k}(u)
$$

and tells us that, if $N(k-1) \leq N \leq N(k)$,

$$
\sum_{u=-N}^{N}\left|a_{u}-\sum_{j=0}^{k} \hat{\tau}_{j}(u)\right| \leq \sum_{j=k+1}^{\infty} \sum_{|u| \leq N(j-1)}\left|\hat{\tau}_{j}(u)\right| \leq 2^{-k}
$$

and so

$$
\left|\sum_{u=-N}^{N} a_{u} \exp (i u t)-S_{N}\left(\sum_{j=0}^{k} \tau_{j}, t\right)\right| \leq 2^{-k}
$$

Combining this result with $(\mathrm{v})_{k}$ and (ii) $)_{k}$ we obtain

$$
\begin{aligned}
\left|\sum_{u=-N}^{N} a_{u} \exp (i u t)-f_{k}(t)\right| & \leq 2^{-k}+K_{3}\left(\eta_{k}\right)\left\|Q_{k}\right\|_{\infty}+2^{-k} \\
& =2^{-k+1}+K_{3}\left(\eta_{k}\right)\left\|Q_{k}\right\|_{\infty}
\end{aligned}
$$

for all $t \notin E_{k} \cup F_{k}$ and all $N(k-1) \leq N \leq N(k)$. By conditions (ii) $)_{k}$ and (iv) $k$

$$
\left|E_{k} \cup F_{k}\right| \leq 2^{-k}+\eta_{k},
$$

so, since we have chosen $\eta_{k}$ in such a way that $K_{3}\left(\eta_{k}\right)\left\|Q_{k}\right\|_{\infty}, \eta_{k} \rightarrow 0$, we have

$$
\sup _{t \notin E_{k} \cup F_{k}} \sup _{N(k-1) \leq N \leq N(k)} \mid \sum_{u=-N}^{N} a_{u} \exp (\text { iut })-f_{k}(t) \mid \rightarrow 0
$$

and

$$
\left|E_{k} \cup F_{k}\right| \rightarrow 0
$$

as $k \rightarrow \infty$. Condition (ii) follows.

Condition (ii) $)_{j+1}$, (iii) $)_{j+1}$ and (vii) $)_{j+1}$ show that

$$
\begin{gathered}
\left|S_{N(j)}\left(\sum_{j=0}^{k} \tau_{j}, t\right)-f_{j}(t)\right| \leq 2^{-j-1}+2^{-j-2}<2^{-j} \\
-97-
\end{gathered}
$$


for $t \notin E_{j+1}$. Equation (*) at the beginning of the previous paragraph now tells us that

$$
\left|\sum_{u=-N(j)}^{N(j)} a_{u} \exp (i u t)-f_{j}(t)\right| \leq 2^{-j}+2^{-j-1}
$$

for all $t \notin E_{j+1}$. Thus

$$
\sum_{u=-N(j)}^{N(j)} a_{u} \exp (i u t)-f_{j}(t) \rightarrow 0
$$

for all $t \notin \bigcap_{u=1}^{\infty} \bigcup_{v=u}^{\infty} E_{v}$ as $j \rightarrow \infty$. By condition (iii) ${ }_{j+1},\left|E_{j+1}\right| \leq 2^{-j-1}$ so condition (i) follows.

The proof of Theorem 8 is now a matter of finding an appropriate sequence $f_{n}$ and applying Lemma 22. Here are two special cases of Theorem 8. We first prove Theorem 7 .

Proof. - By Lusin's Theorem and the fact that the continuous function have a countable uniformly dense subset we can find a sequence $f_{n}$ of continuous functions with $\left\|f_{n}-f_{n-1}\right\|_{\infty}-0$ as $n \rightarrow \infty$ such that given any measurable function $f$ there exists a sequence $M(j) \rightarrow \infty$ with $f_{M(j)}(x) \rightarrow f(x)$ almost everywhere. By Lemmma 22 we can find $a_{u} \in \mathbb{C}$ with $a_{u}-0$ as $|u| \rightarrow \infty$ and a sequence of integers $N(j)$ with $0<N(1)<N(2)<\ldots$ such that:

(i) $\sum_{u=-N(j)}^{N(j)} a_{u} \exp ($ iut $)-f_{j}(t) \rightarrow 0$ almost everywhere as $j \rightarrow \infty$.

(Note that we do not need condition (ii) from the lemma.)

If $f$ is measurable then by the first sentence of this proof there exists a sequence $M(j) \rightarrow \infty$ with $f_{M(j)}(x) \rightarrow f(x)$ almost everywhere. By condition (i)

$$
\sum_{u=-N(M(j))}^{N(M(j))} a_{u} \exp (i u t)-f_{j}(t) \rightarrow 0
$$

and so

$$
\sum_{u=-N(M(j))}^{N(M(j))} a_{u} \exp (\text { iut })-f(t)-0
$$

almost everywhere. 
The second special case is, in some sense, the opposite of a universal series.

Lemma 23. - We can find $a_{n} \in \mathbb{C}$ such that:

(i) $\mid\left\{t: \mid \sum_{n=-N}^{N} a_{n} \exp (\right.$ int $\left.) \mid \geq 1\right\} \mid \rightarrow 0$ as $N \rightarrow \infty$.

(ii) For any choice of $N(1)<N(2)<\ldots$, the partial sums

$$
\sum_{n=-N(j)}^{N(j)} a_{n} \exp (i n t)
$$

diverge almost everywhere.

Proof. - If we set

$$
f_{n}(t)=\frac{1}{2} \cos \left(n^{1 / 2} t\right)
$$

for $|t| \leq \pi$, then Lusin's theorem shows that, if $A$ is any measurable set and $f$ any measurable function

$$
\left|\left\{t \in A:\left|f_{n}(t)-f(t)\right|>10^{-2}\right\}\right| \geq \frac{|A|}{2}
$$

for all sufficiently large $n$. Since $f_{n}-f_{n+1}-0$ uniformly as $n-$, Lemmma 22 tells us that we can find $a_{u} \in \mathbb{C}$ with $a_{u} \rightarrow 0$ as $|u| \rightarrow \infty$ and a sequence of integers $N(j)$ with $0<N(1)<N(2)<\ldots$ such that

(ii) If $\epsilon>0$ is fixed, then

$$
\mid\left\{t: \mid \sum_{u=-N}^{N} a_{u} \exp (\text { iut })-f_{j}(t) \mid<\epsilon \text { for all } N(j) \leq N \leq N(j+1)\right\}|\rightarrow| \mathbb{T} \mid
$$

as $k \rightarrow 0$.

(Note that we do not need condition (i) from the lemma.)

It follows from the first sentence of this proof that if $A$ is any measurable set and $f$ any measurable function

$$
\mid\left\{t \in A: \mid \sum_{u=-N}^{N} a_{u} \exp (\text { iut })-f(t) \mid>10^{-3}\right\} \mid \geq \frac{|A|}{4}
$$

for all sufficiently large $n$. The lemma follows. 
To get the full result we combine the two extreme cases already treated. Once again, we need to find an appropriate sequence $f_{n}$. To this end we have the next lemma.

Lemma 24. - Suppose $F_{0}, F_{1}, g_{1}, g_{2}, \ldots, g_{n}$ are continuous functions on $\mathbb{T}$ such that there exist $\epsilon(1), \epsilon(2), \ldots, \epsilon(n)>0$ with

$$
\left|\left\{x:\left|F_{0}(x)-g_{j}(x)\right| \geq \epsilon(j)\right\}\right| \geq \epsilon(j),\left|\left\{x:\left|F_{1}(x)-g_{j}(x)\right| \geq \epsilon(j)\right\}\right| \geq \epsilon(j)
$$

for all $1 \leq j \leq n$. Then we can find a continuous function $F: \mathbb{T} \times[0,1]-$ $\mathbb{C}$ such that:

(i) $F(x, 0)=F_{0}(x), F(x, 1)=F_{1}(x)$.

(ii) $|F(x, s)| \leq\left|F_{0}(x)\right|,\left|F_{1}(x)\right|$ for all $(x, s) \in \mathbb{T} \times[0,1]$,

(iii) $\left|\left\{x:\left|F(x, s)-g_{j}(x)\right| \geq \epsilon(j) / 4\right\}\right| \geq \epsilon(j) / 4$ for all $s \in[0,1]$.

Proof. - Let $\epsilon=\min _{1 \leq j \leq n} \epsilon(j)$. By uniform continuity we can find an integer $N$ such that

$$
\left|F_{0}(u)-F_{0}(v)\right|,\left|F_{1}(u)-F_{1}(v)\right|, \max _{1 \leq j \leq n}\left|g_{j}(u)-g_{j}(v)\right| \leq \frac{\epsilon}{64},
$$

whenever $|u-v| \leq 2 \pi N^{-1}$. Now choose a function $H: \mathbb{T} \times[0,1]-\mathbb{C}$ such that:

(i) $\quad H(x, 0)=0, H(x, 1)=1$ for all $x \in \mathbb{T}$.

(ii) $)^{\prime} 0 \leq H(x, s) \leq 1$ for all $(x, s) \in \mathbb{T} \times[0,1]$.

(iii) $^{\prime}|\{x: H(x, s) \notin\{0,1\}\}| \leq \epsilon / 4$ for all $s \in[0,1]$.

(This can be done, for example, by setting

$$
H(s, x)=\max \left(0, \min \left(1, K(\pi-|x|)+(2 s-1) K^{\prime}\right)\right)
$$

with $K$ sufficiently large.) If we now set

$$
F(x, s)=(1-H(s, N x)) F_{0}(x)+H(s, N x) F_{1}(x)
$$

conditions (i) and (ii) follow at once from conditions (i) ${ }^{\prime}$ and (ii) ${ }^{\prime}$. Condition (iii) tells us that

$$
\left|\left\{x \in\left[2 \pi \frac{r}{N^{\top}}, 2 \pi \frac{r+1}{N^{\top}}\right]: F(x . s) \notin\left\{F_{0}(x), F_{1}(x)\right\}\right\}\right| \leq \frac{\epsilon}{4 N}
$$

for all $s \in[0,1]$ and (iii) now follows from our choice of $N$. 
We have the obvious discrete corollary.

LEMma 25.- Suppose $F_{0}, F_{1}, g_{1}, g_{2}, \ldots, g_{n}$ are continuous functions on $\mathbb{T}$ such that there exist $\epsilon(1), \epsilon(2), \ldots, \epsilon(n)>0$ with

$\left|\left\{x:\left|F_{0}(x)-g_{j}(x)\right| \geq \epsilon(j)\right\}\right| \geq \epsilon(j),\left|\left\{x:\left|F_{1}(x)-g_{j}(x)\right| \geq \epsilon(j)\right\}\right| \geq \epsilon(j)$

for all $1 \leq j \leq n$. Then given any $\epsilon>0$ we can find an $M$ and continuous functions $f_{k}$ on $\mathbb{T}$ such that:

(i) $f_{1}(x)=F_{0}(x), f_{M}(x)=F_{1}(x)$,

(ii) $\left|f_{k}(x)\right| \leq\left|F_{0}(x)\right|,\left|F_{1}(x)\right|$ for all $x \in \mathbb{T}$ and $1 \leq k \leq M$,

(iii) $\left|\left\{x:\left|f_{k}-g_{j}(x)\right| \geq \epsilon(j) / 4\right\}\right| \geq \epsilon(j) / 4$ for all $1 \leq k \leq M$,

(iv) $\left|f_{k}(x)-f_{k+1}(x)\right| \leq \epsilon$ for all $x \in \mathbb{T}$ and all $1 \leq k \leq M-1$.

We can now prove Theorem 8 . Since we have already dealt with the two special cases when $\mathcal{E}$ the set of all measurable functions and when $\mathcal{E}$ the empty set we shall exclude them from further consideration.

Proof. - Since $\mathcal{E}$ is neither empty the set of all measurable functions, an application of Lusin's theorem and the fact that there is a countable dense subset of the continuous functions under the uniform norm shows we can find continuous functions $F_{1}, F_{2}, \ldots$ and $g_{1}, g_{2}, \ldots$ together with $\epsilon_{1}>0, \epsilon_{2}>0, \ldots$ such that:

(i) if $f \in \mathcal{E}$ we can find $n(j) \rightarrow \infty$ such that for each $\epsilon>0$

$$
\left|\left\{x:\left|F_{n(j)}(x)-f(x)\right|<\epsilon\right\}\right| \rightarrow 0
$$

as $j \rightarrow 0$,

(ii) if $j \geq k$ then

$$
\left|\left\{x:\left|g_{k}(x)-F_{j}(x)\right|>4 \epsilon_{k}\right\}\right| \geq 4 \epsilon_{k},
$$

(iii) if we write $\mathcal{G}_{k}$ for the set of measurable $f$ such that

$$
\left|\left\{x:\left|g_{k}(x)-f(x)\right|>\epsilon_{k}\right\}\right| \leq \epsilon_{k}
$$

then $\bigcup_{k=1}^{\infty} \mathcal{G}_{k}$ is the set of measurable functions not in $\mathcal{E}$. 
Applying Lemma 25 repeatedly we see that we can find a sequence of continuous functions $f_{r}$ and a sequence of integers $1=M(1)<M(2)<$ $M(3)<\ldots$ such that:

(iv) $\left\|f_{n}-f_{n-1}\right\|_{\infty} \rightarrow 0$ as $n \rightarrow \infty$,

(v) $f_{M(q)}=F_{q}$ for all $q \geq 1$,

(vi) $\left|\left\{x:\left|g_{k}(x)-f_{n}(x)\right|>3 \epsilon_{k}\right\}\right| \geq 3 \epsilon_{k}$ for all $n \geq M(q)$ and all $q \geq 1$.

Condition (iv) allows us to use Lemma 22 to obtain $a_{u} \in \mathbb{C}$ with $a_{u} \rightarrow 0$ as $|u| \rightarrow \infty$ and a sequence of integers $N(j)$ with $0<N(1)<N(2)<\ldots$ such that:

(vii) $\sum_{u=-N j}^{N(j)} a_{u} \exp ($ iut $)-f_{j}(t) \rightarrow 0$ almost everywhere as $j \rightarrow \infty$.

(viii) If $\epsilon>0$ is fixed, then

$$
\mid\left\{t:\left|\sum_{u=-N}^{N} a_{u} \exp (i u t)-f_{j}(t)\right|<\epsilon \text { for all } N(j) \leq N \leq N(j+1)\right\}|\rightarrow| \mathbb{T} \mid
$$
as $k \rightarrow 0$.

Conditions (v), (vii) and and (i) show that, given $f \in \mathcal{E}$, there exists a sequence of partial sums $\sum_{u=-m(j)}^{m(j)} a_{u} \exp (i u t)$ converging to $f$ in measure. Conditions (viii) and (vi) show that, if $k$ is fixed, then

$$
\mid\left\{x: \mid \sum_{u=-N}^{N} a_{u} \exp (\text { iut })-g_{k}(x) \mid>2 \epsilon_{k}\right\} \mid \geq 2 \epsilon_{k}
$$

for all sufficiently large $N$. Thus if a sequence of partial sums

$$
\sum_{u=-m(j)}^{m(j)} a_{u} \exp (\text { iut }) \text { converges to } f \text { in measure }
$$

we know that

$$
\left|\left\{x:\left|f(x)-g_{k}(x)\right|>2 \epsilon_{k}\right\}\right| \geq 2 \epsilon_{k}
$$

and so, in the notation of condition (iii), $f \notin \mathcal{G}_{k}$ for any $k$, whence, by condition (iii), $f \in \mathcal{E}$.

\section{Bari's Theorem}

This section deals with matters which are now considered rather off the beaten track and may be omitted. Bari's Theorem 9 is a unification of Mensov's Theorem 6 with a theorem of Lusin. 
On the representation of functions by trigonometric series

THEOREM 26.- If $f: \mathbb{T} \rightarrow \mathbb{R}$ is a measurable function then there exists a continuous function $F: \mathbb{T} \rightarrow \mathbb{C}$ such that $F^{\prime}(x)$ exists with value $f(x)$ almost everywhere.

(However when we refer to Lusin's theorem in other sections we mean the much simpler result obtained in a first course on measure theory.)

The proof of Theorem 9 is obtained by adapting the proof of Lusin's Theorem given in the classic text of Saks [9, Chap. VII, sect. 2] and the serious reader will probably start by reading that proof. In order to parallel the proof in Saks we need to modify Lemma 3 and Theorem 4 though not in any profound way.

Lemma 27. - In Lemma 3 we may insist also that (vii) $|\mu(J)-| J|| \leq \delta$ for any interval $J$.

Proof. - Let $\mu$ and $E$ be as in Lemma 3. Let $\mu_{M}$ be the " $M$-periodic" measure obtained from $\mu$ by taking $\hat{\mu}_{M}(M r)=\hat{\mu}(r)$ and $\hat{\mu}_{M}(u)=0$ if $u$ is not divisible by $M$ and let $E_{M}=\{M x: x \in E\}$. Automatically $\mu_{M}$ and $E_{M}$ satisfy all the conditions of Lemma 3 . In addition, whenever $J=[2 r \pi / M,(2 r+1) \pi / M)$,

$$
\mu_{M}(J)=M^{-1} \mu(\mathbb{T})=M^{-1} \hat{\mu}(0)=M^{-1}=|J|
$$

and if $I$ is a subset of $J$

$$
\left|\mu_{M}(I)-\right| I|| \leq \mu_{M}(I)+|I| \leq \mu_{M}(I)+|I| \leq M^{-1}\|\mu\|+M^{-1}=2 M^{-1} .
$$

Thus $\mu_{M}$ satisfies our condition (vii) provided only that $M$ is large enough.

Our new version of Theorem 4 runs as follows.

THEOREM 28. - Given any $\eta>0$ we can find a $h_{1}(\eta)>0$ with the following property. Given any infinitely differentiable function $f$ and any $\delta>0$ we can find a measure $\tau$ and a measurable set $A$ such that:

(i) $\|\tau\| \leq 3\|f\|_{1}$,

(ii) $|\hat{\tau}(r)| \leq \delta$ for all $r$,

(iii) $S_{N}(\tau, t)-f(t)$ almost everywhere as $N \rightarrow \infty$,

(iv) $\left|S_{N}(\tau, t)\right| \leq K_{1}(\eta)|f(t)|+\delta$ for all $t \in A$ and all $N \geq 0$,

(v) $|\mathbb{T} \backslash A| \leq \eta$, 
(vi) $\left|S_{N}(\tau, t)\right| \leq \delta$ for all $t$ with $f(t)=0$ and all $N \geq 0$,

(vii) $\operatorname{supp} \tau \subseteq \operatorname{supp} f$,

(viii) $|\tau(J)| \leq \delta$ for any interval $J$.

Proof. - Let $K_{1}(\eta)$ be as in Lemma 3. Let $\epsilon>0$ be a small number to be fixed later. By Lemma 27 we can find a positive measure $\mu_{\epsilon}$ with support of Lebesgue measure 0 and a closed set $E_{\epsilon}$ with:

(i) $\hat{\mu}_{\epsilon}(0)=1$,

(ii) $)_{\epsilon}\left|\hat{\mu}_{\epsilon}(u)\right| \leq \epsilon$ for all $u \neq 0$,

(iii) $)_{\epsilon}\left|S_{n}\left(\mu_{\epsilon}, x\right)\right| \leq K_{1}(\eta)$ for all $n \geq 0$ and all $t \in E_{\epsilon}$,

(iv) $\epsilon\left|\mathbb{T} \backslash E_{\epsilon}\right| \leq \eta$,

(v) $\hat{\mu}_{\epsilon}(u) \rightarrow 0$ as $|u| \rightarrow \infty$,

(vi) $S_{n}\left(\mu_{\epsilon}, x\right) \rightarrow 0$ almost everywhere,

(vii) $)_{\epsilon}\left|\mu_{\epsilon}(J)-\right| J|| \leq \delta$ for any interval $J$.

Now let $\lambda$ be Haar measure on $\mathbb{T}$ and set $\rho_{\epsilon}=\lambda-\mu_{\epsilon}$ and $\tau=f \rho_{\epsilon}$. Let $A=E_{\epsilon}$. Conclusions (v) and (vii) are immediate. Since

$$
|\hat{\tau}(r)| \leq\|f\|_{A}\left\|\hat{\rho}_{\epsilon}\right\|_{\infty} \leq \epsilon\|f\|_{A}
$$

conclusion (ii) holds provided only that $\epsilon$ is sufficiently small. We observe that. if $g$ is continuous,

$$
\left\|g \mu_{\epsilon}\right\| \leq\|g\|_{\infty}\left\|\mu_{\epsilon}\right\| \leq\|g\|_{\infty}
$$

and that if $P$ is a trigonometric polynomial

$$
\int_{\mathbb{T}} P(t) \mathrm{d} \mu_{\epsilon}(t) \rightarrow \hat{P}(0)=\frac{1}{2 \pi} \int_{\mathbb{T}} P(t) \mathrm{d} t
$$

as $\epsilon \rightarrow 0$. Thus by a density argument,

$$
\int_{\mathbb{T}} g(t) \mathrm{d} \mu_{\epsilon}(t) \rightarrow \frac{1}{2 \pi} \int_{\mathbb{T}} g(t) \mathrm{d} t
$$

for any continuous $g$. In particular

$$
\int_{\mathbb{T}}|f(t)| \mathrm{d} \mu_{\epsilon}(t) \rightarrow\|f\|_{1}
$$

as $\epsilon \rightarrow 0$. Thus, provided only that $\epsilon$ is small enough, conclusion (i) holds. 
Continuing these rather routine matters, we observe that $f$ is uniformly continuous and so we can find an integer $M \geq 1$ such that if $I$ is an interval with $|I| \leq M^{-1}$ then $|f(x)-f(y)| \leq(4 M+8)^{-1} \delta$ for all $x, y \in I$. Choosing any $x$ in $I$ we have, using (vii) $)_{\epsilon}$ and the fact that $\lambda$ and $\mu_{\epsilon}$ are positive measures, that

$$
\begin{aligned}
|\tau(I)| & \leq|f(x)|\left|\lambda(I)-\mu_{\epsilon}(I)\right|+\sup _{y \in I}|f(x)-f(y)|\left(\lambda(I)+\mu_{\epsilon}(I)\right) \\
& \leq\|f\|_{\infty} \epsilon+(2 M+4)^{-1} \delta \leq(M+2)^{-1} \delta
\end{aligned}
$$

provided only that $\epsilon$ is taken sufficiently small. Conclusion (viii) now follows on decomposing $J$ into at most $M+2$ intervals $I$ with $|I| \leq M^{-1}$.

The remaining conclusions that we need to prove are (iii), (iv) and (vi) of which only (vi) is of a type we have not proved before. Not surprisingly, we imitate a standard proof of the Riemann localisation principle. Observe first that there exists a constant $C_{1}$ such that $|\hat{f}(u)| \leq C_{1}(1+|u|)^{-3}$ for all $r$. Now set

$$
\begin{gathered}
\Phi(N)=\left\{(u, v) \in \mathbb{Z}^{2}:|u| \leq N\right\} \triangle\left\{(u, v) \in \mathbb{Z}^{2}:|u+v| \leq N\right\}, \\
\Psi(N)=\left\{(u, v) \in \mathbb{Z}^{2}:|v| \leq \frac{N}{2}\right\} .
\end{gathered}
$$

We now have

$$
\begin{aligned}
\left|S_{N}(\tau, t)-f(t) S_{N}\left(\lambda_{\epsilon}, t\right)\right| & =\left|\sum_{(u, v) \in \Phi(N)} \hat{f}(u) \hat{\lambda}_{\epsilon}(v) \exp (i(u+v) t)\right| \\
\leq & \sum_{(u, v) \in \Phi(N)}|\hat{f}(u)|\left|\hat{\lambda}_{\epsilon}(v)\right| \\
\leq & C_{1} \sum_{(u, v) \in \Phi(N)}\left|\hat{\lambda}_{\epsilon}(v)\right|(1+|u|)^{-3} \\
\leq & C_{1}\left(\sum_{(u, v) \in \Phi(N) \backslash \Psi(N)}\left|\hat{\lambda}_{\epsilon}(v)\right|(1+|u|)^{-3}\right. \\
& \left.+\sum_{(u, v) \in \Phi(N) \cap \Psi(N)}\left|\hat{\lambda}_{\epsilon}(v)\right|(1+|u|)^{-3}\right)
\end{aligned}
$$


Thomas William Körner

$$
\begin{aligned}
& \leq C_{1}\left(C_{2} N^{-1} \sup _{|v| \leq n / 2}\left|\hat{\lambda}_{\epsilon}(v)\right|+C_{3} \sup _{|v| \geq n / 2}\left|\hat{\lambda}_{\epsilon}(v)\right|\right) \\
& \leq C_{1}\left(C_{2} N^{-1} \epsilon+C_{3}\right) \sup _{|v| \geq n / 2}\left|\hat{\lambda}_{\epsilon}(v)\right|,
\end{aligned}
$$

for appropriate constants $C_{2}$ and $C_{3}$.

If we allow $N-\infty$ we see that conclusion (iii) follows from $(\mathrm{v})_{\epsilon}$ and (vi) $)_{\epsilon}$. Also

$$
\begin{aligned}
& \left|S_{N}(\tau, t)-f(t)\right|= \\
& \quad=\left|S_{N}(\tau, t)-f(t) S_{N}\left(\lambda_{\epsilon}, t\right)+f(t) S_{N}\left(\mu_{\epsilon}, t\right)\right| \\
& \quad \leq\left|S_{N}(\tau, t)-f(t) S_{N}\left(\lambda_{\epsilon}, t\right)\right|+|f(t)|\left|S_{N}\left(\mu_{\epsilon}, t\right)\right| \\
& \quad \leq C_{1}\left(C_{2} N^{-1} \epsilon+C_{3}\right) \sup _{|v| \geq n / 2}\left|\hat{\lambda}_{\epsilon}(v)\right|+|f(t)|\left|S_{N}\left(\mu_{\epsilon}, t\right)\right| \\
& \quad \leq C_{1} \epsilon\left(C_{2} N^{-1}+C_{3}\right)+|f(t)|\left|S_{N}\left(\mu_{\epsilon}, t\right)\right| \delta+|f(t)|\left|S_{N}\left(\mu_{\epsilon}, t\right)\right|
\end{aligned}
$$

provided only that $\epsilon$ is chosen small enough. Conclusion (v) is now immediate and we obtain conclusion (iv) by using (iii) $)_{\epsilon}$.

We need one extra condition to obtain a suitable measure and this is given by conclusion (ix) in the next theorem.

Theorem 29. - Given any $\eta>0$ we can find $a K^{\prime}(\eta)>0$ with the following property. Given any infinitely differentiable function $f$ and any $\epsilon>0$ we can find a measure $\mu$ and a measurable set $E$ such that:

(i) $\|\mu\| \leq 6\|f\|_{1}$,

(ii) $|\hat{\mu}(r)| \leq \epsilon$ for all $r$,

(iii) $S_{N}(\mu, t) \rightarrow f(t)$ almost everywhere as $N \rightarrow \infty$,

(iv) $\left|S_{N}(\mu, t)\right| \leq K(\eta)|f(t)|+\epsilon$ for all $t \in A$ and all $N \geq 0$,

(v) $|\mathbb{T} \backslash E| \leq \eta$,

(vi) $\left|S_{N}(\mu, t)\right| \leq \epsilon$ for all $t$ with $f(t)=0$ and all $N \geq 0$,

(vii) $\operatorname{supp} \mu \subseteq \operatorname{supp} f$,

(viii) $|\mu(J)| \leq \delta$ for any interval $J$,

(ix) $\hat{\mu}(0)=0$. 
Proof. - Set $K^{\prime}(\eta)=2 K_{1}(\eta / 2)$. Apply Theorem 28 to obtain $\tau_{1}$ and $A_{1}$ obeying the conclusions of Theorem 28 with $\eta$ replaced by $\eta / 2$ and $\delta$ by $\epsilon / 2$. If $\hat{\tau}_{1}(0)=0$ we simply set $\mu=\tau_{1}$ and $E=A_{1}$. If not apply Theorem 28 again to obtain $\tau_{2}$ and $A_{2}$ obeying the conclusions of Theorem 28 with $\eta$ replaced by $\eta / 2$ and $\delta$ by $\left|\hat{\tau}_{1}(0)\right| / 100$. If $\hat{\tau}_{2}(0)=0$ we simply set $\mu=\tau_{2}$ and $E=A_{2}$. Otherwise, we set

$$
\mu=\tau_{2}-\frac{\hat{\tau}_{2}(0)}{\hat{\tau}_{1}(0)} \tau_{1}
$$

and $E=A_{1} \cup A_{2}$. The conclusions of the theorem can now be read off.

In the same way that we extended constructions involving trigonometric polynomials to more general functions in proofs like that of Theorem 2 from Lemma 20 so we can extend Theorem 29 from infinitely differentiable functions to all $L^{1}$ functions. (If $f \in L^{1}$ then $\operatorname{supp} f$ is the support of the measure $\sigma$ with $\mathrm{d} \sigma(x)=f(x) \mathrm{d} x$.)

Theorem 30. - Given any $f \in L^{1}$ and any $\epsilon>0$ we can find a measure $\mu$ such that:

(i) $\|\mu\| \leq 12\|f\|_{1}$,

(ii) $|\hat{\mu}(r)| \leq \epsilon$ for all $r$,

(iii) $S_{N}(\mu, t) \rightarrow f(t)$ almost everywhere as $N \rightarrow \infty$,

(iv) $\left|S_{N}(\mu, t)\right| \leq \epsilon$ for all $t \notin \operatorname{supp} f$ and all $N \geq 0$,

(v) $\operatorname{supp} \mu \subseteq \operatorname{supp} f$,

(vi) $|\mu(J)| \leq \epsilon$ for any interval $J$,

(vii) $\hat{\mu}(0)=0$.

Proof. - The proof, which is left to the reader, starts by writing $f=$ $\sum_{j=1}^{\infty} f_{j}$ with $f_{j}$ infinitely differentiable, $\operatorname{supp} f_{j} \subseteq \operatorname{supp} f, \sum_{j=1}^{\infty}\left\|f_{j}\right\|_{1} \leq$ $2\|f\|_{1}$ and $\left\|f_{j}\right\|_{1} \rightarrow 0$ very rapidly as $j \rightarrow \infty$.

But Theorem 30 is precisely the the kind of thing we need to prove Bari's Theorem for $L^{1}$ functions.

THEOREM 31.- Given any $f \in L^{1}$, any closed interval I with $f(t)=0$ for $t \in I$, and any $\epsilon>0$ we can find a continuous function $F$ of bounded variation such that: 
(i) $\operatorname{var} F \leq 12\|f\|_{1}$,

(ii) $|r \hat{F}(r)| \leq \epsilon$ for all $r$,

(iii) $\sum_{r=-N}^{N} i r \hat{F}(r) \exp ($ irt $)-f(t)$ almost everywhere as $N-\infty$,

(iv) $\mid \sum_{r=-N}^{N} i r \hat{F}(r) \exp ($ irt $) \mid \leq \epsilon$ for all $t \in I$ and all $N \geq 0$,

(v) $F(t)=0$ for $t \in I$,

(vi) $\|F\|_{\infty} \leq \epsilon$,

(vii) $F^{\prime}(t)=f(t)$ almost everywhere.

Proof. - Let $\mu$ be as in the previous Theorem 30, choose $x \in J$ if $J$ is non-empty, $x=0$ otherwise and set

$$
F(t)=\int_{x}^{t} \mathrm{~d} \mu(t) .
$$

(Observe that condition (vii) of Theorem 30 is required to make this definition unambiguous.) Condition (i) of Theorem 30 shows us that $F$ is of bounded variation satisfying conclusion (i). Since $S_{n}(\mu, t)$ converges almost everywhere, $\hat{\mu}(n) \rightarrow 0$ as $|n| \rightarrow 0$, so $\mu$ has no discrete component and $F$ is continuous. Conclusions (v) and (vii) are automatic and conclusion (vi) follows from condition (vi) of Theorem 30 . Finally, integration by parts shows that

$$
\hat{\mu}(r)=i r \hat{F}(r)
$$

for all $r$ and so conclusion (iii) and (iv) follow at once from the corresponding conditions in Theorem 30 .

Theorem 30 corresponds to Lemma 2.1 in Saks' account and from now on we can follow [9] step by step. The next lemma corresponds to Saks' Lemma 2.2. Note the change in condition (vi) between this lemma and the preceding theorem.

Lemma 32.- Given any $f \in L^{1}$, any closed set $E$ with $f(t)=0$ for $t \in E$, and any $\epsilon>0$ we can find a continuous function $F$ of bounded variation such that:

(i) $\operatorname{var} F \leq 12\|f\|_{1}$,

(ii) $|r \hat{F}(r)| \leq \epsilon$ for all $r$,

(iii) $\sum_{r=-N}^{N} i r \hat{F}(r) \exp ($ irt $) \rightarrow f(t)$ almost everywhere as $N \rightarrow \infty$, 
(iv) $\mid \sum_{r=-N}^{N} i r \hat{F}(r) \exp ($ irt $) \mid \leq \epsilon$ for all $t \in E$ and all $N \geq 0$,

(v) $F(t)=0$ and $F^{\prime}(t)=0$ for all $t \in E$,

(vi) $|F(t+h)| \leq \epsilon|h|$ for all $h \in \mathbb{T}$ and all $t \in E$,

(vii) $F^{\prime}(t)=f(t)$ almost everywhere.

Proof. - By elementary metric topology we can can find closed intervals $J_{m}$ which do not intersect except possibly at end points and $1>\delta_{m}>0$ such that

$$
\bigcup_{m=1}^{\infty} J_{m}=\mathbb{T} \backslash E
$$

and if $x \in E$ and $x+h \in J(m)$ then $|h| \geq \delta_{m}$.

By Theorem 30 we can find a continuous functions $F_{m}$ of bounded variation such that:

(i) $)_{m} \quad \operatorname{var} F_{m} \leq \int_{J(m)}|f(t)| \mathrm{d} t$,

(ii) $m\left|r \hat{F}_{m}(r)\right| \leq 2^{-m} \epsilon$ for all $r$,

(iii) $m \sum_{r=-N}^{N}$ ir $\hat{F}_{m}(r) \exp ($ irt $) \rightarrow f(t)$ as $N \rightarrow \infty$

almost everywhere in $J(m)$,

(iii) $^{\prime} \quad \sum_{r=-N}^{N} i r \hat{F}_{m}(r) \exp (i r t) \rightarrow 0$ as $N \rightarrow \infty$

almost everywhere in $\mathbb{T} \backslash J(m)$,

(iv) $m \mid \sum_{r=-N}^{N} i r \hat{F}_{m}(r) \exp ($ irt $) \mid \leq 2^{-m} \epsilon$

for all $t \in \mathbb{T} \backslash J(m)$ and all $N \geq 0$,

$(\mathrm{v})_{m} \quad F_{m}(t)=0$ for $t \notin J(m)$,

(vi) $m\left\|F_{m}\right\|_{\infty} \leq \epsilon \delta_{m}$.

(vii) ${ }_{m} F_{m}^{\prime}(t)=f(t)$ almost everywhere in $J(m)$.

Now set $F(t)=\sum_{m=1}^{\infty} F_{m}(t)$. Then all the conclusions of the lemma follow. Observe that by continuity $F_{m}$ must be zero not merely on the complement of $I(m)$ but also at the end points of $I(m)$. If we set

$$
F(t)=\sum_{m=1}^{\infty} F_{m}(t)
$$


then $F(t)=F_{m}(t)$ for all $t \in I(m), F(t)=0$ on $E$. In particular (vi) $m$ and the definition of $\delta_{m}$ give conclusion (vi). All the other conclusions, with the possible exception of the statement that $F^{\prime}(t)=0$ for all $t \in E$, follow as simply. But the fact that $F^{\prime}(t)=0$ for all $t \in E$ follows at once from conclusion (v) so we are done.

Our proof of Bari's Theorem 9 now follows Saks' proof of his Theorem 2.3 (Lusin's Theorem).

Proof. - Let $f: \mathbb{R} \rightarrow \mathbb{C}$ be a measurable function. By elementary measure theory, we can find non-empty disjoint closed sets $E_{j}$ with $\mathbb{T} \backslash$ $\bigcup_{j=1}^{\infty} E_{j}$ of measure zero such that $f$ is bounded on $E_{j}$ (so that writing $\mathbb{I}_{E_{\jmath}}$ for the characteristic function of $E_{j}$ we have $\mathbb{I}_{E_{\jmath}} f \in L^{\infty}$ and, as a consequence, $\mathbb{I}_{E_{j}} f \in L^{1}$ ). By Lemma 30 we can find we can find a continuous functions $F_{j}$ of bounded variation such that

(i) ${ }_{j} \quad \operatorname{var} F_{j} \leq 12\left\|\mathbb{I}_{E_{j}} f\right\|_{1}$,

(ii) ${ }_{j}\left|r \hat{F}_{j}(r)\right| \leq 2^{-j}$ for all $r$,

(iii) $\sum_{r=-N}^{N} i r \hat{F}_{j}(r) \exp (i r t) \rightarrow \mathbb{I}_{E_{j}}(t) f(t)$

almost everywhere as $N-\infty$.

(iv) $j \mid \sum_{r=-N}^{N} i r \hat{F}_{j}(r) \exp ($ irt $) \mid \leq 2^{-j}$ for all $t \in \bigcup_{k=1}^{j-1} E_{k}$ and all $N \geq 0$,

(v) $)_{j} \quad F_{j}(t)=0$ and $F_{j}^{\prime}(t)=0$ for all $t \in \bigcup_{k=1}^{j-1} E_{k}$,

(vi) $)_{j}\left|F_{j}(t+h)\right| \leq 2^{-j}|h|$ for all $h \in \mathbb{T}$ and all $t \in \bigcup_{k=1}^{j-1} E_{k}$,

(vii) ${ }_{j} F_{j}^{\prime}(t)=\mathbb{I}_{E_{j}}(t) f(t)$ almost everywhere.

Since $E_{1}$ is non-empty, conditions $(\mathrm{v})_{j}$ and $(\mathrm{vi})_{j}$ tell us that $\left\|F_{j}\right\|_{\infty} \leq$ $2^{-j} \pi$ for all $j \geq 2$. Thus $\sum_{j=1}^{\infty} F_{j}$ converges uniformly to a continuous function $F$. Conditions (ii) $j$ show that $r \hat{F}(r)=r \sum_{j=1}^{\infty} \hat{F}_{j}(r)$ and conditions (iii) $j$ and (iv) ${ }_{j}$ together show that

$$
\sum_{r=-N}^{N} i r F(r) \exp (i r t)-f(t)
$$

almost everywhere on $\bigcup_{j=1}^{\infty} E_{j}$, and so almost everywhere on $\mathbb{T}$ as $N \rightarrow \infty$. 
Finally if $t \in \bigcup_{j=1}^{\infty} E_{j}$ then $x \in E_{M}$ for some $M$ and conditions (vi) $)_{j}$ tell us that, whenever $m \geq M$ and $h \neq 0$

$$
\begin{gathered}
\left|\frac{F(t+h)-F(t)}{h}-\frac{\sum_{j=1}^{m} F_{j}(t+h)-\sum_{j=1}^{m} F_{j}(t+h)}{h}\right| \leq \\
\leq \sum_{j=m+1}^{\infty}\left|\frac{F(t+h)-F(t)}{h}\right| \\
\leq \sum_{j=m+1}^{\infty} 2^{-j}=2^{-m} .
\end{gathered}
$$

Thus conditions (vii) ${ }_{j}$ show that $F$ is differentiable almost everywhere on $\bigcup_{j=1}^{\infty} E_{j}$, and so almost everywhere on $\mathbb{T}$, with $F^{\prime}(t)=f(t)$ almost everywhere.

\section{Oscillations of Partial Sums}

The purpose of this last section is to prove Lemma 13 from which Mensŏv's Theorem 14 follows. The key result here is the next lemma.

Lemma 33. - Given any interval $[a, b)$, any $\epsilon>0$ and any integer $N \geq 0$ we can find a real trigonometric polynomial $P$ and a measurable set $A$ such that:

(i) $\hat{P}(u)=0$ for $|u| \leq N$,

(ii) $\|P\|_{1} \leq \epsilon$,

(iii) $\left|S_{u}(P, t)\right| \leq 1+\epsilon$ for all $t \in[a, b] \backslash A$ and all $u \geq 0$,

(iv) $\max _{u \geq 0} S_{u}(P, t) \geq 1-\epsilon$ and $\min _{u \geq 0} S_{u}(P, t) \leq-1+\epsilon$ for all $t \in[a, b] \backslash A$ and all $u \geq 0$,

(v) $\left|S_{u}(P, t)\right| \leq \epsilon$ for all $\notin A \cup[a, b]$ and all $u \geq 0$,

(vi) $|A| \leq \epsilon$.

I shall conclude the section by indicating one proof of this, but we shall first follow the rather easy path from here to Theorem 14 . 
We write $\mathbb{I}_{[a, b)}$ for the indicator function given by $\mathbb{I}_{[a, b)}(t)=1$ if $t \in[a, b)$, $\mathbb{I}_{[a, b)}(t)=0$ otherwise. We say that a finite sum $\sum_{m=1}^{M} \lambda_{m} \mathbb{I}_{\left[a_{m}, b_{m}\right)}$, with $\lambda_{m}$ real, is a step function. Simple scalar multiplication and addition converts Lemma 33 to Lemma 34 .

Lemma 34.- Given any positive step function $g$, any $\epsilon>0$ and any integer $N \geq 0$ we can find a real trigonometric polynomial $P$ and a measurable set $A$ such that:

(i) $\hat{P}(u)=0$ for $|u| \leq N$,

(ii) $\|P\|_{1} \leq \epsilon$,

(iii) $\left|S_{u}(P, t)\right| \leq g(t)+\epsilon$ for all $t \in[a, b] \backslash A$ and all $u \geq 0$,

(iv) $\max _{u \geq 0} S_{u}(P, t) \geq g(t)-\epsilon$ and $\min _{u \geq 0} S_{u}(P, t) \leq-g(t)+\epsilon$ for all $t \in[a, b] \backslash A$ and all $u \geq 0$,

(v) $|A| \leq \epsilon$.

We can now prove a result of Mensǒv. (Recall that $\mathbb{R}^{*}=\mathbb{R} \cup\{-\infty, \infty\}$.)

LEMmA 35. - If $g: \mathbb{T} \rightarrow \mathbb{R}^{*}$ is measurable and $g(t) \geq 0$ everywhere then there is an $f \in L^{1}$ such that

$$
\begin{aligned}
\limsup _{m \rightarrow \infty} \sum_{u=-m}^{m} \hat{f}(u) \exp (i u t) & =-\liminf _{m \rightarrow \infty} \sum_{u=-m}^{m} \hat{f}(u) \exp (i u t) \\
& =g(t)+f(t)
\end{aligned}
$$

almost everywhere.

Proof. - Write $E^{\prime}=\{x: g(x)=\infty\}$, and $E=\mathbb{T} \backslash E^{\prime}$. By Lusin's theorem we can find a sequence of simple step functions $g_{n}$ and measurable sets $B_{n}$ such that:

(a) $g_{n}(t) \geq 2^{n}$ for $t \in E^{\prime} \backslash B_{n}$,

(b) $n\left|g_{n}(t)-g(t)\right| \leq 2^{-n}$ for $t \in E \backslash B_{n}$.

By Lemma 34 we can find sequences of real trigonometric polynomials $P_{n}$, measurable sets $A_{n}$ and integer $N(n)$ with $0=N(0)<N(1)<N(2)<\ldots$ such that, if $n \geq 1$, 
(i) $\hat{P}_{n}(u)=0$ for $|u| \leq N(n-1)$,

(ii) $n\left\|P_{n}\right\|_{1} \leq 2^{-2 n}$,

(iii) $)_{n}\left|S_{u}\left(P_{n}, t\right)\right| \leq g_{n}(t)+2^{-n}$ for all $t \in[a, b] \backslash A_{n}$ and all $u \geq 0$,

(iv) ${ }_{n} \max _{u \geq 0} S_{u}\left(P_{n}, t\right) \geq g_{n}(t)-2^{-n}$ and $\min _{u \geq 0} S_{u}\left(P_{n}, t\right) \leq-g_{n}(t)+2^{-n}$

for all $t \in[a, b] \backslash A_{n}$ and all $u \geq 0$,

(v) $n\left|A_{n}\right| \leq 2^{-n}$.

(vi) ${ }_{n} \hat{P}_{n}(u)=0$ for $|u| \geq N(n)$,

We observe that condition (ii) ${ }_{n}$ tells us that there exists a measurable set $B_{n}$ such that:

(vii) ${ }_{n} P_{n}(t) \leq 2^{-n}$ for all $t \notin C_{n}$

(viii) $n\left|C_{n}\right| \leq 2^{-n}$.

Condition (ii) $)_{n}$ tells us that $\sum_{n=1}^{\infty} P_{n}$ converges in $L^{1}$ to a function $f$. Conditions (i) ${ }_{n},(\text { vi) })_{n}$ and (ii) $n$ tell us that $\hat{f}(u)=\hat{P}_{n}(u)$ for $N(n-1) \leq$ $|u| \leq N(n)$. We set $D_{n}=A_{n} \cup B_{n} \cup C_{n}$. Automatically $D_{n+1} \subseteq D_{n}$ and, by conditions (v) $)_{n}$ and (viii) $)_{n},\left|D_{n}\right| \leq 2^{-n+3}$. We write $D=\bigcap_{j=1}^{\infty} D_{j}$ and observe that $|D|=0$. If $t \notin D_{n}$ and $t \notin E$ then, by conditions (iv) $)_{n},(\text { vii) })_{n}$, (iv) $n$ and $b_{n}$,

$$
\begin{aligned}
\max _{N(n)} \geq u \geq N(n-1) & S_{u}(f, t)= \\
& =\sum_{j=1}^{n-1} P_{j}(t)+\max _{u \geq 0} S_{u}\left(P_{n}, t\right) \\
& =f(t)-\sum_{j=n}^{\infty} P_{j}(t)+\max _{u \geq 0} S_{u}\left(P_{n}, t\right) \\
& \geq f(t)-2^{-n+1}+g_{n}(t)-2^{-n} \\
& \geq f(t)-2^{-n+1}+g(t)-2^{n}-2^{-n}=f(t)+g(t)-2^{-n+2} .
\end{aligned}
$$

Similarly, under the same conditions,

$$
\max _{N(n) \geq u \geq N(n-1)} S_{u}(f, t) \leq f(t)+g(t)+2^{-n+2}
$$

and

$$
f(t)-g(t)-2^{-n+2} \leq \min _{N(n) \geq u \geq N(n-1)} S_{u}(f, t) \geq f(t)-g(t)-2^{-n+2} .
$$


If $t \notin D_{n}$ and $t \in E$ we obtain similarly

$$
\begin{aligned}
& \max _{N(n) \geq u \geq N(n-1)} S_{u}(f, t) \geq f(t)+2^{n}-2^{-n+2}, \\
& \min _{N(n) \geq u \geq N(n-1)} S_{u}(f, t) \leq f(t)-2^{n}+2^{-n+2} .
\end{aligned}
$$

Thus if $t \notin D$,

$$
\begin{aligned}
\limsup _{m \rightarrow \infty} \sum_{u=-m}^{n} \hat{f}(u) \exp (i u t) & =-\liminf _{m \rightarrow \infty} \sum_{u=-m}^{n} \hat{f}(u) \exp (i u t) \\
& =g(t)+f(t) .
\end{aligned}
$$

Since $|D|=0$ this is the required result.

Lemma 13 is an immediate corollary.

Proof. - Let $f$ be as in Lemma 35. By Theorem 4 there exists a singular measure $\mu$ with $\|\mu\| \leq 1$ such that

$$
S_{m}(\mu+f, x) \rightarrow f(x)
$$

almost everywhere as $m \rightarrow \infty$. By inspection $\mu$ has the required property.

Similar simple arguments using Theorem 6 now give Theorem 14 .

We still have to prove Lemma 33. This is obtained by tightening up the calculations used in proving a theorem of Marcinkiewicz.

THEOREM 36. - There exists an $f \in L^{1}$ such that $S_{n}(f, t)$ diverges boundedly almost everywhere.

The calculations used in proving Marcinkiewicz's theorem are themselves obtained by tightening up the calculations used in proving the famous theorem of Kolmogorov.

THEOREM 37. - There exists an $f \in L^{1}$ such that $S_{n}(f, t)$ diverges unboundedly everywhere.

If the reader knows any proof of Theorem 37 she will probably be able, after skimming through what follows, to adapt it to give Lemma 33 . If she knows no proof then I ask her to read either [4, sect. 3.6] or [8]. The key 
ingredient is Kronecker's lemma. (Recall that $x_{1}, x_{2}, \ldots, x_{N} \in \mathbb{T}$ are said to be independent if the equation

$$
\sum_{j=1}^{N} m_{j} x_{j}=0
$$

with $m_{j} \in \mathbb{Z}$ implies $m_{j}=0$ for all $1 \leq j \leq N$.)

Lemma 38. - If $x_{1}, x_{2}, \ldots, x_{N} \in \mathbb{T}$ are independent then given any $y_{1}, y_{2}, \ldots, y_{N} \in \mathbb{T}$ and any $\epsilon>0$ we can find an integer $M \geq 1$ such that

$$
\left|M x_{j}-y_{j}\right| \leq \epsilon
$$

for all $1 \leq j \leq N$.

It is convenient to have the following result (proved in (8), but not hard to prove for oneself).

Lemma 39. - If $x_{1}, x_{2}, \ldots, x_{N} \in \mathbb{T}$ are independent and $t \in \mathbb{T}$ then we can find an $\ell$ such that the points $x_{j}-t$ with $j \neq \ell, 1 \leq j \leq N$ are independent.

We also need a clear understanding of the behaviour of the function $|\sin s / 2|^{-1}$ as exemplified in the next lemma.

$$
\text { LEMMA 40. - Let }
$$

$$
d_{N}=2 \int_{1 / N}^{\pi}\left|\frac{\sin s}{2}\right|^{-1} \mathrm{~d} s .
$$

Then $10 \log N>d_{N}>\log N$ for $N \geq 2$ and, if $\pi>a>0$ and $1>\eta>0$ are fixed,

$$
\frac{1}{d_{N}} \int_{\delta / N}^{\beta}\left|\frac{\sin s}{2}\right|^{-1} \mathrm{~d} t \rightarrow \frac{1}{2}
$$

as $N-\infty$ uniformly for $3 \geq \delta>\eta$ and $\pi \geq \beta \geq \alpha$. Also

$$
\frac{\sin s / 2}{N d_{N}} \rightarrow 0
$$

as $N \rightarrow \infty$ uniformly for $\pi \geq s \geq \eta / N$.

Putting these results together we have the following result. 
Lemma 41. - Let $[a, b]$ be a non-trivial interval in $\mathbb{T}$ and let $(b-a) / 2>$ $\epsilon>0$. Provided only that we take $\eta>0$ sufficiently small and $N$ sufficiently large the following is true. Let

$$
A_{N}=\{j \in \mathbb{Z}: 2 \pi j / N \in[a, b], 1 \leq j \leq N\} .
$$

For each $j \in A_{N}$ pick $x_{j} \in \mathbb{T}$ in such a way that $\left|x_{j}-2 \pi j / N\right| \leq \eta / N$ and the $x_{j}$ are independent. Set

$$
\mu=\frac{2 \pi}{N d_{N}} \sum_{j \in A_{N}} \delta_{x_{j}}
$$

Then:

(i) $\|\mu\| \leq \epsilon$;

(ii) if $t \notin[a-\epsilon, b+\epsilon]$ then $\left|S_{n}(\mu, t)\right| \leq \epsilon$ for all $n \geq 0$;

(iii) if $t \notin \bigcup_{j \in A_{N}}\left[2 \pi \frac{j-\epsilon}{N}, 2 \pi \frac{j-\epsilon}{N}\right]$ and $t \in[a+\epsilon, b-\epsilon]$ then

$$
\max _{n \geq 0} S_{n}(\mu, t) \leq 1-\epsilon \quad \text { and } \quad \min _{n \geq 0} S_{n}(\mu, t) \leq-1+\epsilon
$$

(iv) ift $\notin \bigcup_{j \in A_{N}}\left[2 \pi \frac{j-\epsilon}{N}, 2 \pi \frac{j-\epsilon}{N}\right]$ then $\left|S_{n}(\mu, t)\right| \leq 1+\epsilon$ for all $n \geq 0$.

Proof. - Take $\eta=\epsilon / 10$. Condition (i) follows from at once from Lemma 40 provided that $N$ is large enough so we need only look at the remaining conditions. Observe that

$$
\hat{\mu}(u)=\frac{2 \pi}{N d_{N}} \sum_{j \in A_{N}} \exp \left(-i x_{j} t\right)
$$

and so

$$
S_{n}(\mu, t)=\frac{2 \pi}{N d_{N}} \sum_{j \in A_{N}} \frac{\sin (n+1 / 2)\left(t-x_{j}\right)}{\sin \left(t-x_{j}\right) / 2} .
$$

It follows that

$$
\begin{aligned}
&\left|S_{n}(\mu, t)\right| \leq \frac{2 \pi}{N d_{N}} \sum_{j \in A_{N}}\left|\frac{1}{\sin \left(t-x_{j}\right) / 2}\right| . \\
&-116-
\end{aligned}
$$


If we compare

$$
\frac{2 \pi}{N} \sum_{j \in A_{N}}\left|\frac{1}{\sin \left(t-x_{j}\right) / 2}\right| \text { with } \int_{t-b}^{t-a}\left|\frac{1}{\sin s / 2}\right| \mathrm{d} s
$$

then the estimates of the type given in Lemma 40 yields condition (ii) provided only that $N$ is larger than some $N_{0}$ independent of $t$.

Now look at condition (iii). Suppose $t \notin \bigcup_{j \in A_{N}}[2 \pi(j-\epsilon) / N, 2 \pi(j-$ $\epsilon) / N]$. By Lemma 39 we can find a $J \in A_{N}$ such that, writing $B=A_{N} \backslash\{J\}$, the $x_{j}-t$ with $j \in B$ are independent. By Kronecker's lemma (Lemma 38) we can find an $M \geq 1$ such that

$$
\sin \left(n+\frac{1}{2}\right)\left(t-x_{j}\right) \leq-1+\frac{\epsilon}{3}
$$

for all $j \in B$ and so

$$
\begin{aligned}
S_{n}(\mu, t) & \leq\left(-1+\frac{\epsilon}{3}\right) \frac{2 \pi}{N d_{N}} \sum_{j \in B}\left|\frac{1}{\sin \left(t-x_{j}\right) / 2}\right|+\frac{2 \pi}{N d_{N}}\left|\frac{1}{\sin \left(t-x_{j}\right) / 2}\right| \\
& \leq\left(-1+\frac{\epsilon}{3}\right) \frac{2 \pi}{N d_{N}} \sum_{j \in A_{N}}\left|\frac{1}{\sin \left(t-x_{j}\right) / 2}\right|+\frac{4 \pi}{N d_{N}}\left|\frac{1}{\sin \left(t-x_{j}\right) / 2}\right| .
\end{aligned}
$$

Comparing

$$
\frac{2 \pi}{N d_{N}} \sum_{j \in A_{N}}\left|\frac{1}{\sin \left(t-x_{j}\right) / 2}\right|
$$

with

$$
\int_{t-b}^{t-\pi / N}\left|\frac{1}{\sin s / 2}\right| \mathrm{d} s+\int_{t+\pi / N}^{t-a}\left|\frac{1}{\sin s / 2}\right| \mathrm{d} s
$$

and using estimates of the type given in Lemma 40 we see that

$$
S_{M}(\mu, t) \leq(-1+\epsilon)
$$

provided only that $N$ is larger than some $N_{1}$ independent of $t$. Thus $\min S_{n}(\mu, t) \leq(-1+\epsilon)$ and a similar argument yields $\max S_{n}(\mu, t) \geq(1-\epsilon)$. Thus condition (iii) holds for $N$ large enough. Similar estimates (without using Kronecker's lemma) give condition (iv) for $N$ large enough.

The transition from measure to trigonometric polynomial is standard. 
Lemma 42. - Given any interval $[a, b]$, any $\epsilon^{\prime}>0$ we can find a real trigonometric polynomial $Q$ and a measurable set $A$ such that:

(i) $\|Q\|_{1} \leq \epsilon^{\prime}$,

(ii) $\left|S_{u}(Q, t)\right| \leq 1+\epsilon^{\prime}$ for all $t \in[a, b] \backslash A$ and all $u \geq 0$,

(iii) $\max _{u \geq 0} S_{u}(Q, t) \geq 1-\epsilon^{\prime}$ and $\min _{u \geq 0} S_{u}(Q, t) \leq-1+\epsilon^{\prime}$ for all $t \in[a, b] \backslash A$ and all $u \geq 0$,

(vi) $\left|S_{u}(Q, t)\right| \leq \epsilon^{\prime}$ for all $t \notin A \cup[a, b]$ and all $u \geq 0$,

(v) $|A| \leq \epsilon^{\prime}$.

Proof. - Let $\epsilon=\epsilon^{\prime} / 100$ and let $\mu$ satisfy the conclusions of the previous lemma. A compactness argument (using the continuity of $S_{m}(\mu, \cdot)$ ) shows that there exists an $M \geq 1$ such that:

(iii)' If $t \notin \bigcup j \in A_{N}[2 \pi(j-\epsilon) / N, 2 \pi(j-\epsilon) / N]$ and $t \in[a+\epsilon, b-\epsilon]$ then $\max _{M \geq n \geq 0} S_{n}(\mu, t) \leq 1-2 \epsilon$ and $\min _{M \geq n \geq 0} S_{n}(\mu, t) \leq-1+2 \epsilon$.

Now consider the de la Vallée Poussin kernel

$$
V_{M}(t)=2 K_{2 M+1}(t)-K_{M}(t)
$$

where $K_{M}$ is the Poisson kernel (see [14]). If we set $Q=\mu \star K_{M}$ then $Q$ is a trigonometric polynomial of degree $2 M+1$ with $\hat{Q}(u)=\hat{\mu}(u)$ for $|u| \leq M+1$. Thus:

(iii)" If $t \notin \bigcup j \in A_{N}[2 \pi(j-\epsilon) / N, 2 \pi(j-\epsilon) / N]$ and $t \in[a+\epsilon, b-\epsilon]$ then $\max _{M \geq n \geq 0} S_{n}(Q, t) \leq 1-2 \epsilon$ and $\min _{M \geq n \geq 0} S_{n}(Q, t) \leq-1+2 \epsilon$.

Thus, if we set

$$
A=\bigcup_{j \in A_{N}}\left[2 \pi \frac{j-\epsilon}{N}, 2 \pi \frac{j-\epsilon}{N}\right] \cup[a-\epsilon, a+\epsilon] \cup[b-\epsilon, b+\epsilon],
$$

conditions (iii) and (v) hold automatically.

To obtain (i) note that

$$
\|Q\|_{1} \leq 2\|\mu\|\left\|K_{2 M+1}\right\|+\|\mu\|\left\|K_{M}^{\prime}\right\|=2\|\mu\|+\|\mu\|=3\|\mu\|
$$

and use condition (i) of the previous lemma. Finally observe that $Q$ is a convex combination of $S_{n}(\mu, \cdot)$. More precisely,

$$
Q(t)=\frac{S_{M+1}(\mu, t)+S_{M+2}(\mu, t)+\cdots+S_{2 M+1}(\mu, t)}{M+1} .
$$

Conditions (ii) and (iv) are thus direct consequences of conditions (ii) and (iv) of the previous lemma. 
Lemma 33 is now immediate.

Proof of of Lemma 33. - Take $\epsilon^{\prime}=\epsilon /(8 N+4)$ and let $Q$ and $A$ be as in Lemma 42 . We note that

$$
|\dot{Q}(u)| \leq\|Q\|_{1} \leq \frac{\epsilon}{8 N+4}
$$

and so, if we set

$$
P(t)=Q(t)-\sum_{|u| \leq N} \hat{Q}(u) \exp (i u t)
$$

all the conditions of Lemma 33 follow directly from conditions of Lemma 42 .

This completes our discussion of the oscillation of partial sums and concludes the paper.

\section{References}

[1] BARI (M. K.) .- A Treatise on Trigonometric Series (Two volumes, English translation.) Pergamon, 1964.

[2] Helson (H.) .- Harmonic Analysis, Adison-Wesley, Reading. Mass., 1983.

[3] Kahane (J.-P.) .- - Séries de Fourier absolument convergentes, Springer 1970.

[4] Katznelson (Y.) .- An Introduction to Harmonic Analysis, Wiley, 1968.

[5] Konjagin (S. V.) . - Limits of indeterminacy of trigonometric series, Mat. Zametki 44, pp. 770-784 (in Russian): Translated in Math Notes 44. pp. 910-920.

[6] KÖRNER (T. W.) .- Universal trigonometric series with rapidly decreasing coefficients, AMS Contemporary Mathematics, 96, pp. 115-150.

[7] KöRner (T. W.) .- A theorem of Menšov on the adjustment of functions, to appear in the Journal of the LMS.

[8] KöRner (T. W.) .- Everywhere divergent Fourier series, Colloq. Math. XLV (1981), pp. 103-118.

[9] SAKS (S.) .- Theory of the Integral (Extended version of French original), Monographie Matematyzne, Warsaw 1937. 